Supporting information

\title{
A comparison of the rheological and mechanical properties of isotactic, syndiotactic, and heterotactic PLA
}

\author{
Love-Ese Chile, ${ }^{1,2}$ Parisa Mehrkhodavandi ${ }^{1}{ }^{*}$ and Savvas G. Hatzikiriakos ${ }^{2 *}$
}

${ }^{1}$ Department of Chemistry, University of British Columbia, 2036 Main Mall, Vancouver, British Columbia, Canada; ${ }^{2}$ Department of Chemical and Biological Engineering, University of British Columbia, Vancouver, BC, Canada.

\section{Contents}

A. Polymerization and molecular weight data of meso-lactide with catalysts 1 and $2 \ldots \ldots \ldots \ldots . .2$

B. Kinetic investigations and comparison to literature catalysts ......................................

C. Molecular weight polymerization data for various microstructured PLAs .........................5

D. Determination of tacticity and ${ }^{1} \mathrm{H}\left\{{ }^{1} \mathrm{H}\right\}$ NMR spectra of polymers ................................ 6

E. GCMS traces from meso-lactide purification ............................................................... 16

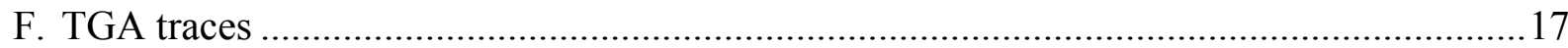

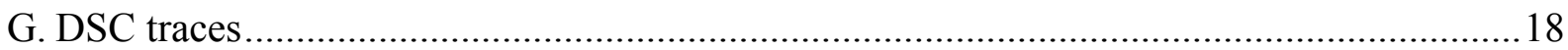

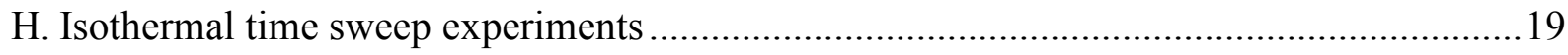

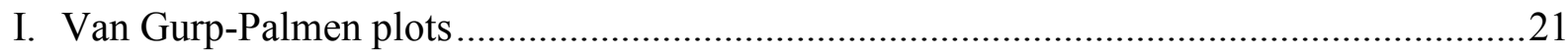

J. Arrhenius plot and determination of activation of flow energy ....................................24

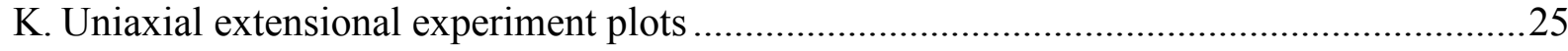

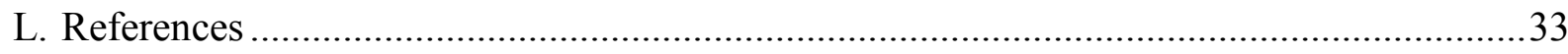




\section{A. Polymerization and molecular weight data of meso-lactide with catalysts 1 and 2}

Table S1. Polymerization of lactide with dinuclear indium complexes $( \pm)-\mathbf{1}$ and $(R R / R R)-\mathbf{1}$.

\begin{tabular}{lllllll}
\hline Entry & Catalyst & {$[\mathrm{LA}] /[$ initiator $]$} & $\begin{array}{l}\mathrm{M}_{\mathrm{n}, \text { theo }} \\
\left(\mathrm{gmol}^{-1}\right)^{b}\end{array}$ & $\begin{array}{l}\mathrm{M}_{\mathrm{n}, \text { expt }} \\
\left(\mathrm{gmol}^{-1}\right)^{c}\end{array}$ & $\mathrm{P}_{s}{ }^{d}$ & $Ð$ \\
\hline $1^{e}$ & $(R R / R R)-1$ & 1000 & 27650 & 30200 & 0.85 & 1.00 \\
2 & $(R R / R R)-1$ & 250 & 35390 & 30160 & 0.85 & 1.02 \\
3 & $(R R / R R)-1$ & 470 & 66390 & 50250 & 0.85 & 1.04 \\
4 & $(R R / R R)-1$ & 870 & 79750 & 73810 & 0.86 & 1.00 \\
5 & $(R R / R R)-1$ & 1500 & 21800 & 203800 & 0.86 & 1.04 \\
6 & $(R R / R R)-1$ & 2000 & 283600 & 275200 & 0.86 & 1.03 \\
7 & $(R R / R R)-1$ & 2400 & 343700 & 299400 & 0.86 & 1.07 \\
8 & $( \pm)-1$ & 250 & 35310 & 44280 & 0.83 & 1.02 \\
9 & $( \pm)-1$ & 490 & 69630 & 72710 & 0.81 & 1.01 \\
10 & $( \pm)-1$ & 990 & 140600 & 126800 & 0.82 & 1.01 \\
11 & $( \pm)-1$ & 1500 & 212700 & 198100 & 0.82 & 1.02 \\
12 & $( \pm)-1$ & 2000 & 281300 & 255200 & 0.83 & 1.03 \\
13 & $( \pm)-1$ & 2500 & 350200 & 218600 & 0.82 & 1.09
\end{tabular}

Reactions were carried out in $\mathrm{CH}_{2} \mathrm{Cl}_{2}, 25{ }^{\circ} \mathrm{C},[1]_{\mathrm{o}} \approx 2.8 \mathrm{mM} ;{ }^{a}$ Conversions calculated from ${ }^{1} \mathrm{H}$

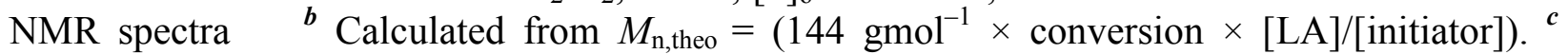
Molecular weights were determined by GPC-LALLS (gel permeation chromatography-low angle laser light scattering) to the polystyrene standard calibration curve via the Mark-Houwink equation in THF at $25^{\circ} \mathrm{C}\left([\eta]=K \mathrm{M}^{a}\right.$, while $[\eta]=$ intrinsic viscosity, $\mathrm{M}=$ molecular weight, and $K$ and $a$ are Mark-Houwink parameters $K=1.832 \times 10-4 \mathrm{dLg}^{-1}$, and $a=0.69 \mathrm{dn} / \mathrm{dc}=0.044$ $\left.\mathrm{mLg}^{-1}\right)$. THF $4 \mathrm{mg} \mathrm{mL}^{-1}$ and flow rate $=0.5 \mathrm{~mL} \mathrm{~min}^{-1} .{ }^{d}$ Calculated from ${ }^{1} \mathrm{H}\left\{{ }^{1} \mathrm{H}\right\} \mathrm{NMR}$ spectra. ${ }^{1}$ ${ }^{e}$ Experiment carried out at $0{ }^{\circ} \mathrm{C}$ for 48 hours. 
Table S2. Polymerization of meso-lactide with dinuclear indium complexes $( \pm)-\mathbf{2}$ and $(R R / R R)$ 2.

\begin{tabular}{|c|c|c|c|c|c|c|}
\hline Entry & Catalyst & [LA]/[initiator] & $\begin{array}{l}\mathrm{M}_{\mathrm{n}, \text { theo }} \\
\left(\mathrm{gmol}^{-1}\right)^{b}\end{array}$ & $\begin{array}{l}\mathrm{M}_{\mathrm{n}, \mathrm{expt}} \\
\left(\mathrm{gmol}^{-1}\right)^{c}\end{array}$ & $\mathrm{P}_{s}^{d}$ & $Ð$ \\
\hline 1 & $(R R / R R)-\mathbf{2}$ & 250 & 35310 & 30190 & 0.77 & 1.02 \\
\hline 2 & $(R R / R R)-\mathbf{2}$ & 490 & 69900 & 48850 & 0.78 & 1.22 \\
\hline 3 & $(R R / R R)-\mathbf{2}$ & 980 & 141300 & 74730 & 0.78 & 1.25 \\
\hline 4 & $(R R / R R)-\mathbf{2}$ & 1400 & 201100 & 102900 & 0.78 & 1.22 \\
\hline 5 & $(R R / R R)-\mathbf{2}$ & 1800 & 259500 & 104500 & 0.78 & 1.18 \\
\hline 6 & $(R R / R R)-\mathbf{2}$ & 2300 & 328000 & 121300 & 0.78 & 1.26 \\
\hline 7 & $( \pm)-2$ & 250 & 35310 & 31640 & 0.77 & 1.22 \\
\hline 8 & $( \pm)-2$ & 490 & 70630 & 52450 & 0.78 & 1.17 \\
\hline 9 & $( \pm)-2$ & 800 & 115300 & 52110 & 0.77 & 1.04 \\
\hline 10 & $( \pm)-2$ & 1300 & 190300 & 109600 & 0.77 & 1.03 \\
\hline 11 & $( \pm)-2$ & 2000 & 282500 & 143100 & 0.77 & 1.07 \\
\hline 12 & $( \pm)-2$ & 2500 & 353100 & 146400 & 0.77 & 1.14 \\
\hline
\end{tabular}

Reactions were carried out in $\mathrm{CH}_{2} \mathrm{Cl}_{2}, 25{ }^{\circ} \mathrm{C},[2]_{\mathrm{o}} \approx 2.7 \mathrm{mM} ;{ }^{a}$ Conversions calculated from ${ }^{\mathrm{I}} \mathrm{H}$

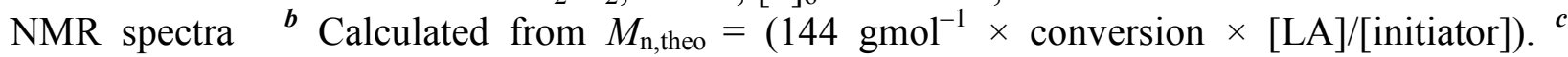
Molecular weights were determined by GPC-LALLS (gel permeation chromatography-low angle laser light scattering) to the polystyrene standard calibration curve via the Mark-Houwink equation in THF at $25^{\circ} \mathrm{C}\left([\eta]=K \mathrm{M}^{a}\right.$, while $[\eta]=$ intrinsic viscosity, $\mathrm{M}=$ molecular weight, and $K$ and $a$ are Mark-Houwink parameters $K=1.832 \times 10-4 \mathrm{dLg}^{-1}$, and $a=0.69 \mathrm{dn} / \mathrm{dc}=0.044$ $\left.\mathrm{mLg}_{1}^{-1}\right)$. THF $4 \mathrm{mg} \mathrm{mL}^{-1}$ and flow rate $=0.5 \mathrm{~mL} \min ^{-1} \cdot{ }^{d}$ Calculated from ${ }^{1} \mathrm{H}\left\{{ }^{1} \mathrm{H}\right\} \mathrm{NMR}$ spectra. 


\section{B. Kinetic investigations and comparison to literature catalysts}

Table S3. Rates of polymerization of lactide isomers with various catalysts.

\begin{tabular}{llllll}
\hline \multirow{2}{*}{ Entry } & Catalyst & \multicolumn{3}{c}{$k_{o b s}\left(\times 10^{-3} \mathrm{~s}^{-1}\right)$ for } & $P_{\mathrm{s}}{ }^{a}$ \\
& & meso-LA & rac-LA & L-LA & \\
\hline 1 & $( \pm)-\mathbf{1}$ & $1.61(0.03)^{b}$ & $1.72(0.16)^{c}$ & $2.98(0.09)^{c}$ & 0.83 \\
2 & $(R R / R R)-\mathbf{1}$ & $0.35(0.5)^{b}$ & $0.62(0.16)^{c}$ & $3.4(0.6)^{c}$ & 0.86 \\
3 & $(S S / S S)-\mathbf{1}$ & $0.31(0.03)^{b}$ & $0.70(0.05)^{c}$ & $0.27(0.04)$ & 0.87 \\
4 & $( \pm)-2$ & $2.52(0.02)^{d}$ & $2.3(0.5)^{e}$ & $2.6(0.5)^{e}$ & 0.78 \\
5 & $(R R / R R)-2$ & $2.24(0.01)^{d}$ & $0.46(0.09)^{e}$ & $2.6(0.5)^{e}$ & 0.79 \\
6 & $\mathbf{3}^{f}$ & $1.36 \times 10^{-2}$ & $1.25 \times 10^{-2}$ & $2.97 \times 10^{-2}$ & 0.70 \\
7 & $\mathbf{4}^{g}$ & $0.77 \times 10^{-1}$ & $2.28 \times 10^{-1}$ & $4.15 \times 10^{-1}$ & 0.57 \\
8 & $\mathbf{5}^{h}$ & $3.3 \times 10^{-2}$ & $7.3 \times 10^{-2}$ & $\mathrm{n} / \mathrm{a}$ & 0.96 \\
9 & $\mathbf{6}^{i}$ & $1.18 \times 10^{-1}$ & $3.94 \times 10^{-2}$ & $4.56 \times 10^{-2}$ & 0.20
\end{tabular}

${ }^{a} P_{\mathrm{s}}$ values shown for meso-LA. ${ }^{b}[\mathrm{LA}]=0.46 \mathrm{M}$. [catalyst] $=0.0023 \mathrm{M}$. Average of three experiments; ${ }^{c}[\mathrm{LA}]=0.46 \mathrm{M}$. [catalyst] $=0.0024 \mathrm{M} ;{ }^{6}{ }^{d}[\mathrm{LA}]=0.36 \mathrm{M}$. [catalyst] $=0.0018 \mathrm{M}$. Average of three experiments ; ${ }^{e}[\mathrm{LA}]=0.45 \mathrm{M}$. [catalyst] $=0.0023 \mathrm{M}^{7}{ }^{b-e}$ Reactions were carried out in an NMR tube at $25^{\circ} \mathrm{C}$ and followed to $90 \%$ conversion. 1,3,5-trimethoxybenzene (TMB) was used as internal standard. The value of $k_{o b s}$ was determined from the slope of $\ln [\mathrm{LA}]$ vs. time, averaged from three experiments. For entries 5-8 see the corresponding references: ${ }^{f}$ Okuda 2011; ${ }^{8}$ Chen and Feijen 2009; ${ }^{9 h}$ Coates $2002 ; ;^{i}$ Okuda 2011. ${ }^{10}$ 


\section{Molecular weight polymerization data for various microstructured PLAs}

Table S4. Polymerization data for various microstructured PLAs used for themorheological experiments

\begin{tabular}{|c|c|c|c|c|}
\hline Entry & Catalyst & $\begin{array}{l}\mathrm{M}_{\mathrm{n}, \operatorname{expt}} \\
\left(\mathrm{gmol}^{-1}\right)^{a}\end{array}$ & Tacticity $^{b}$ & $\bigoplus^{a}$ \\
\hline $1^{c}$ & $\mathrm{Sn}(\mathrm{oct})_{2}$ & 49880 & $P_{\mathrm{r}}=0.56$ & 1.11 \\
\hline $2^{c}$ & $\mathrm{Sn}(\mathrm{oct})_{2}$ & 154600 & $P_{\mathrm{r}}=0.60$ & 1.12 \\
\hline $3^{c}$ & $\mathrm{Sn}(\mathrm{oct})_{2}$ & 248700 & $P_{\mathrm{r}}=0.57$ & 1.14 \\
\hline $4^{c}$ & $\mathrm{Sn}(\mathrm{oct})_{2}$ & 352500 & $P_{\mathrm{r}}=0.55$ & 1.10 \\
\hline $5^{c}$ & $\mathrm{InCl}_{3} / \mathrm{NEt}_{3} / \mathrm{BnOH}$ & 147600 & $P_{\mathrm{r}}=0.95$ & 1.04 \\
\hline $6^{c}$ & $\mathrm{InCl}_{3} / \mathrm{NEt}_{3} / \mathrm{BnOH}$ & 182100 & $P_{\mathrm{r}}=0.96$ & 1.05 \\
\hline $7^{c}$ & $\mathrm{InCl}_{3} / \mathrm{NEt}_{3} / \mathrm{BnOH}$ & 254500 & $P_{\mathrm{r}}=0.95$ & 1.02 \\
\hline $8^{c}$ & $\mathrm{InCl}_{3} / \mathrm{NEt}_{3} / \mathrm{BnOH}$ & 294600 & $P_{\mathrm{r}}=0.94$ & 1.05 \\
\hline $9^{d}$ & $(R R / R R)-\mathbf{1}$ & 129500 & $P_{\mathrm{s}}=0.83$ & 1.01 \\
\hline $10^{d}$ & $(R R / R R)-\mathbf{1}$ & 146700 & $P_{\mathrm{s}}=0.84$ & 1.02 \\
\hline $11^{d}$ & $(R R / R R)-\mathbf{1}$ & 224200 & $P_{\mathrm{s}}=0.83$ & 1.02 \\
\hline $12^{d}$ & $(R R / R R)-\mathbf{1}$ & 278700 & $P_{\mathrm{s}}=0.82$ & 1.01 \\
\hline $13^{d}$ & $(R R / R R)-2$ & 66740 & $P_{\mathrm{m}}=0.73$ & 1.06 \\
\hline $14^{d}$ & $(R R / R R)-2$ & 100400 & $P_{\mathrm{m}}=0.72$ & 1.02 \\
\hline $15^{d}$ & $(R R / R R)-2$ & 103700 & $P_{\mathrm{m}}=0.72$ & 1.08 \\
\hline $16^{d}$ & $(R R / R R)-\mathbf{2}$ & 118600 & $P_{\mathrm{m}}=0.71$ & 1.04 \\
\hline
\end{tabular}

${ }^{a}$ Molecular weights were determined by GPC-LALLS (gel permeation chromatography-low angle laser light scattering) to the polystyrene standard calibration curve via the Mark-Houwink equation in THF at $25^{\circ} \mathrm{C}\left([\eta]=K \mathrm{M}^{a}\right.$, while $[\eta]=$ intrinsic viscosity, $\mathrm{M}=$ molecular weight, and $K$ and $a$ are Mark-Houwink parameters $K=1.832 \times 10-4 \mathrm{dLg}^{-1}$, and $a=0.69 \mathrm{dn} / \mathrm{dc}=0.044$ $\left.\mathrm{mLg}^{-1}\right)$. THF $4 \mathrm{mg} \mathrm{mL}^{-1}$ and flow rate $=0.5 \mathrm{~mL} \mathrm{~min}^{-1} \cdot{ }^{b}$ Calculated from ${ }^{1} \mathrm{H}\left\{{ }^{1} \mathrm{H}\right\} \mathrm{NMR}$ spectra. ${ }^{c}$ Reactions were carried out in toluene at $70{ }^{\circ} \mathrm{C}$. Reactions were carried out in $\mathrm{CH}_{2} \mathrm{Cl}_{2}$ at $25{ }^{\circ} \mathrm{C}$. 


\section{Determination of tacticity and ${ }^{1} \mathrm{H}\left\{{ }^{1} \mathrm{H}\right\}$ NMR spectra of polymers}

Work done in 1992 by Kricheldorf et. al. and continued by Thakur and co-workers, ${ }^{23-27}$ utilized Bernoullian statistics to investigate the different possible four unit tetrad sequences based on the stereochemistry of the starting monomer. The probabilities for various meso-LA tetrads are shown in Table $1 .^{2}$

Table S5. Tetrad probabilities for meso-lactide, based on Bernoullian statistics.

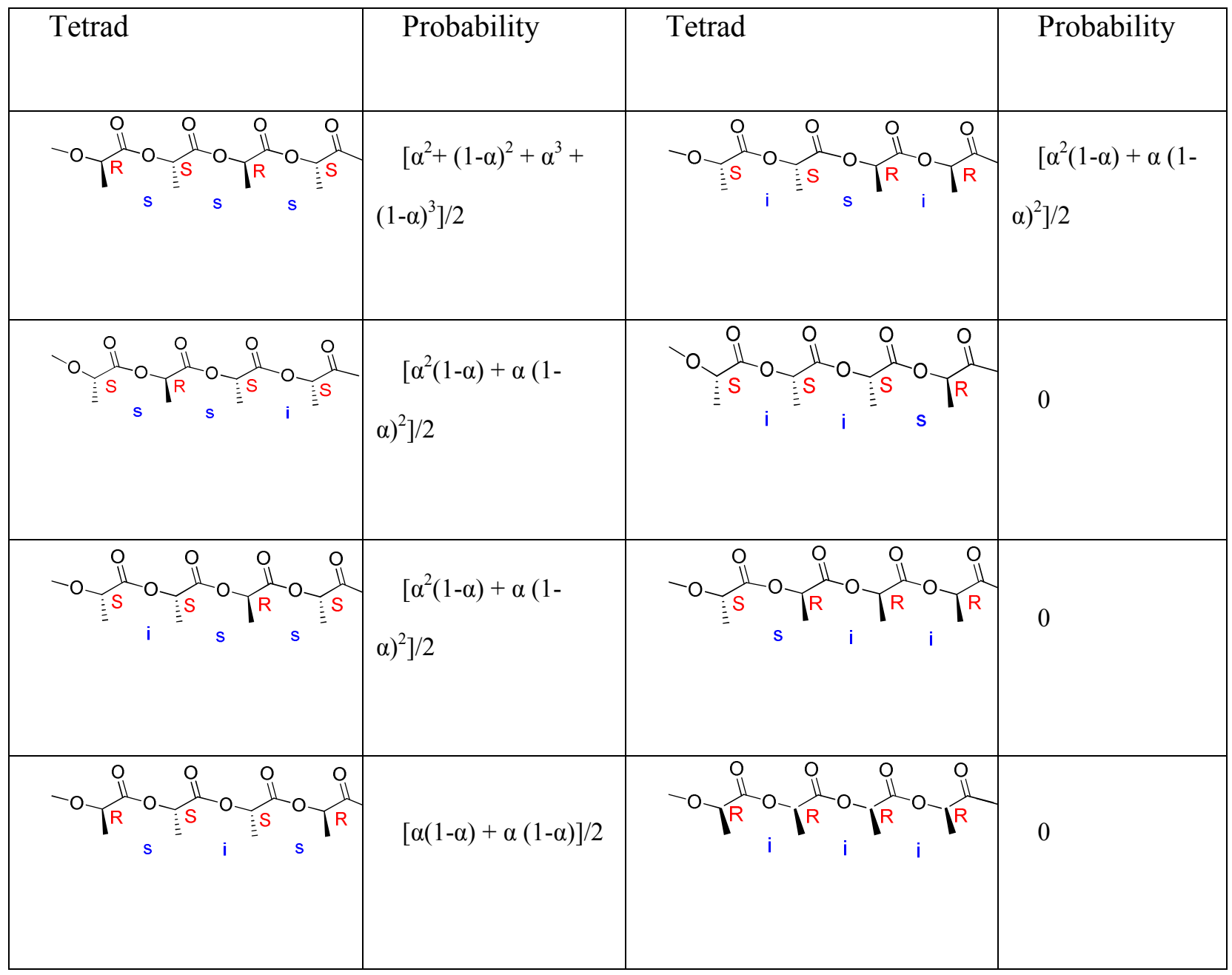


The level of stereocomplexity, or tacticity occurring within a particular polymer chain can be determined by combining the probabilities of observing the various tetrad sequences with integration data obtained from homonuclear decoupled ${ }^{1} \mathrm{H}$ NMR spectra.

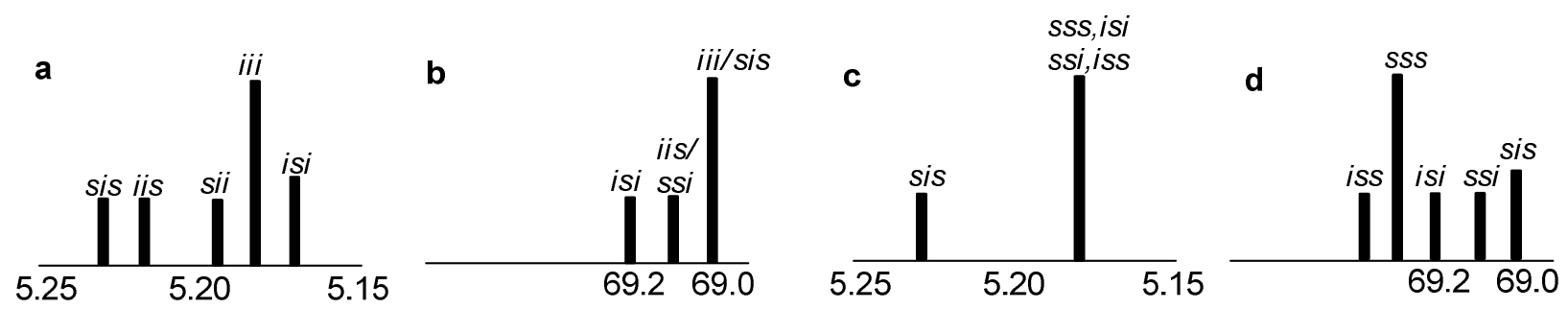

Figure S1. Schematic diagrams of PLA tetrad resonances corresponding to the methane region in (a) homonuclear decoupled ${ }^{1} \mathrm{H}$ NMR of PLA from $\mathrm{rac}$-lactide; (b) ${ }^{13} \mathrm{C}$ NMR of PLA from $\mathrm{rac}$ lactide; (c) homonuclear decoupled ${ }^{1} \mathrm{H}$ NMR of PLA from meso-lactide; (d) ${ }^{13} \mathrm{C}$ NMR mesolactide. Reproduced from Okuda 2011. ${ }^{3}$

$$
\begin{aligned}
& {[s s s]=P_{s}^{2}+\frac{P_{s} P_{i}}{2}} \\
& {[s i s]=\frac{P_{i}^{2}+P_{s} P_{i}}{2}} \\
& {[s s i]=[i s s]=\frac{P_{S} P_{i}}{2}} \\
& {[i s i]=\frac{P_{i}{ }^{2}}{2}}
\end{aligned}
$$

Equation S1. Calculation of $P_{\mathrm{s}}$ values used by Okuda et. al, based on probability of tetrad sequences in PLA from on Bernoullian statistics. ${ }^{3}$

$$
\begin{aligned}
& \alpha=\frac{\mathrm{z}}{(\mathrm{x}+\mathrm{y}+\mathrm{z})} \\
& {[\mathrm{sss}]=1 / 2\left[\alpha^{3}+(1-\alpha)^{3}+\alpha^{2}+(1-\alpha)^{2}\right]} \\
& {[\mathrm{sis}]=\alpha(1-\alpha)} \\
& {[\mathrm{ssi}]=[\mathrm{iss}]=[\mathrm{isi}]=1 / 2\left[\alpha^{2}(1-\alpha)+\alpha(1-\alpha)^{2}\right]} \\
& {[\mathrm{ssi} / \mathrm{iss} / \mathrm{isi}]=3\left(1 / 2\left[\alpha^{2}(1-\alpha)+\alpha(1-\alpha)^{2}\right]\right)} \\
& P_{i}=[\mathrm{sis}] \\
& P_{s}=[\mathrm{sss}]+[\mathrm{ssi} / \mathrm{iss} / \mathrm{isi}]
\end{aligned}
$$

Equation S2. Calculation of $P_{\mathrm{s}}$ values used in this study by Coates et. al. 


$$
\begin{aligned}
\alpha & =\frac{z}{(x+y+z)} \\
& =0.773
\end{aligned}
$$

$$
\begin{aligned}
{[\text { sss }] } & =1 / 2\left[\alpha^{3}+(1-\alpha)^{3}+\alpha^{2}+(1-\alpha)^{2}\right] \\
= & 0.561 \\
P \mathrm{i}= & {[\text { sis }]=\alpha(1-\alpha) } \\
= & 0.175
\end{aligned}
$$

$$
\begin{aligned}
& {[\text { ssi } / \text { iss } / \text { isi }] }=3\left(1 / 2\left[\alpha^{2}(1-\alpha)+\alpha(1-\alpha)^{2}\right]\right) \\
&=0.264 \\
& P s=[\mathrm{sss}]+[\mathrm{ssi} / \mathrm{iss} / \mathrm{isi}] \\
&=0.825
\end{aligned}
$$

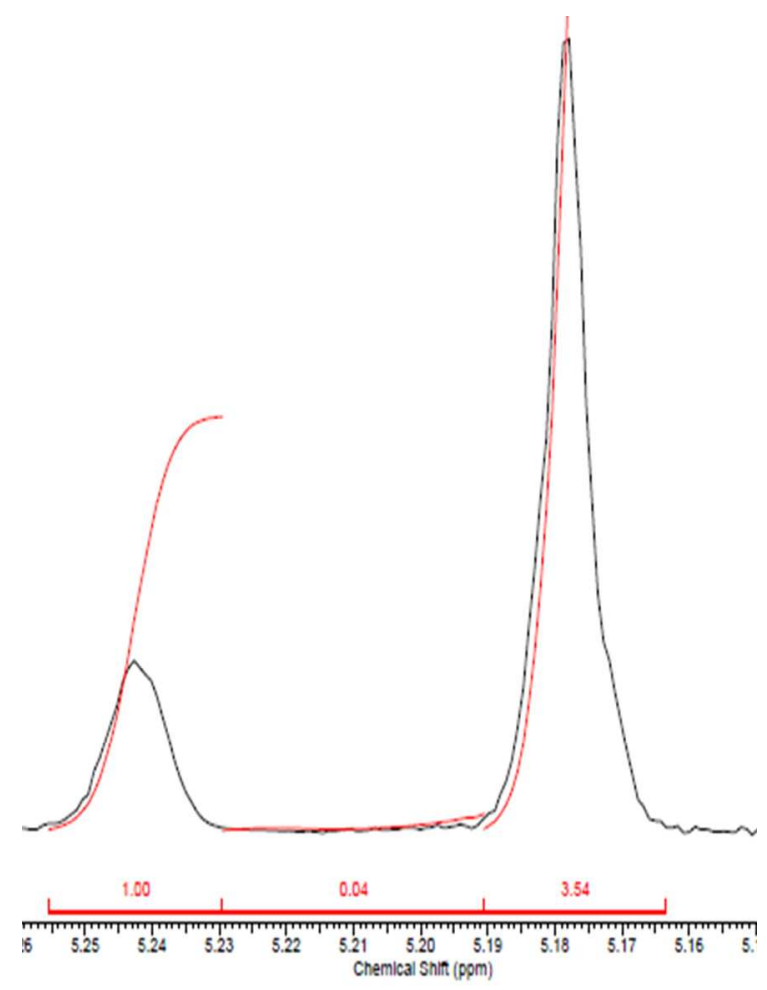

Figure S2. ${ }^{1} \mathrm{H}\left\{{ }^{1} \mathrm{H}\right\}$ NMR spectrum of polymer produced from reaction of ( \pm )-1 with mesolactide and calculation of $P_{s}$ value 


$$
\begin{aligned}
& \alpha=\frac{\mathrm{z}}{(\mathrm{x}+\mathrm{y}+\mathrm{z})} \\
& =0.829 \\
& {[\mathrm{sss}]=1 / 2\left[\alpha^{3}+(1-\alpha)^{3}+\alpha^{2}+(1-\alpha)^{2}\right]} \\
& =0.646 \\
& P \mathrm{i}=[\text { sis }]=\alpha(1-\alpha) \\
& =0.142 \\
& {[\mathrm{ssi} / \mathrm{iss} / \mathrm{isi}]=3\left(1 / 2\left[\alpha^{2}(1-\alpha)+\alpha(1-\alpha)^{2}\right]\right)} \\
& =0.213 \\
& P s=[\mathrm{sss}]+[\mathrm{ssi} / \mathrm{iss} / \mathrm{isi}] \\
& =0.859
\end{aligned}
$$

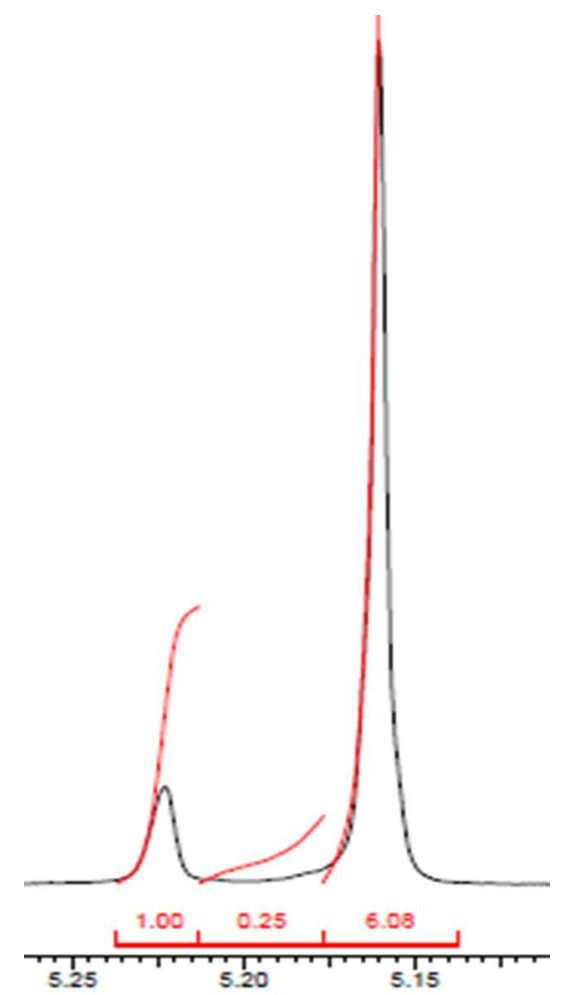

Figure S3. ${ }^{1} \mathrm{H}\left\{{ }^{1} \mathrm{H}\right\}$ NMR spectrum of polymer produced from reaction of $(R R / R R)-\mathbf{1}$ with mesolactide and calculation of $P_{s}$ value 


$$
\begin{aligned}
& \alpha=\frac{z}{(x+y+z)} \\
& =0.662 \\
& {[\mathrm{sss}]=1 / 2\left[\alpha^{3}+(1-\alpha)^{3}+\alpha^{2}+(1-\alpha)^{2}\right]} \\
& =0.441 \\
& P \mathrm{i}=[\mathrm{sis}]=\alpha(1-\alpha) \\
& =0.223 \\
& \text { [ssi/iss } / \text { isi }]=3\left(\frac{1}{2}\left[\alpha^{2}(1-\alpha)+\alpha(1-\alpha)^{2}\right]\right) \\
& =0.336 \\
& P s=[\mathrm{sss}]+[\mathrm{ssi} / \mathrm{iss} / \mathrm{isi}] \\
& =0.777
\end{aligned}
$$

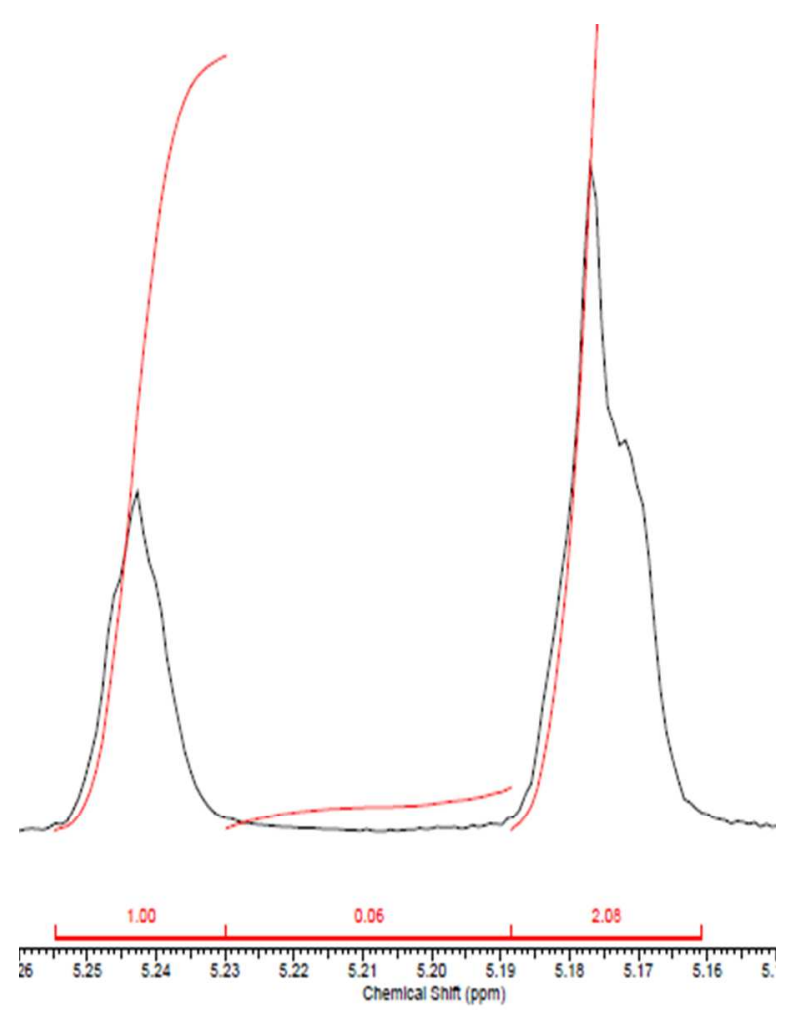

Figure S4. ${ }^{1} \mathrm{H}\left\{{ }^{1} \mathrm{H}\right\}$ NMR spectrum of polymer produced from reaction of $( \pm)-2$ with mesolactide and calculation of $P_{s}$ value 


$$
\begin{aligned}
& \alpha=\frac{\mathrm{z}}{(\mathrm{x}+\mathrm{y}+\mathrm{z})} \\
& =0.707 \\
& {[\mathrm{sss}]=1 / 2\left[\alpha^{3}+(1-\alpha)^{3}+\alpha^{2}+(1-\alpha)^{2}\right]} \\
& =0.482 \\
& P \mathrm{i}=[\text { sis }]=\alpha(1-\alpha) \\
& =0.207 \\
& {[\text { ssi } / \text { iss } / \text { isi }]=3\left(1 / 2\left[\alpha^{2}(1-\alpha)+\alpha(1-\alpha)^{2}\right]\right)} \\
& =0.312 \\
& P s=[\mathrm{sss}]+[\mathrm{ssi} / \mathrm{iss} / \mathrm{isi}] \\
& =0.794
\end{aligned}
$$

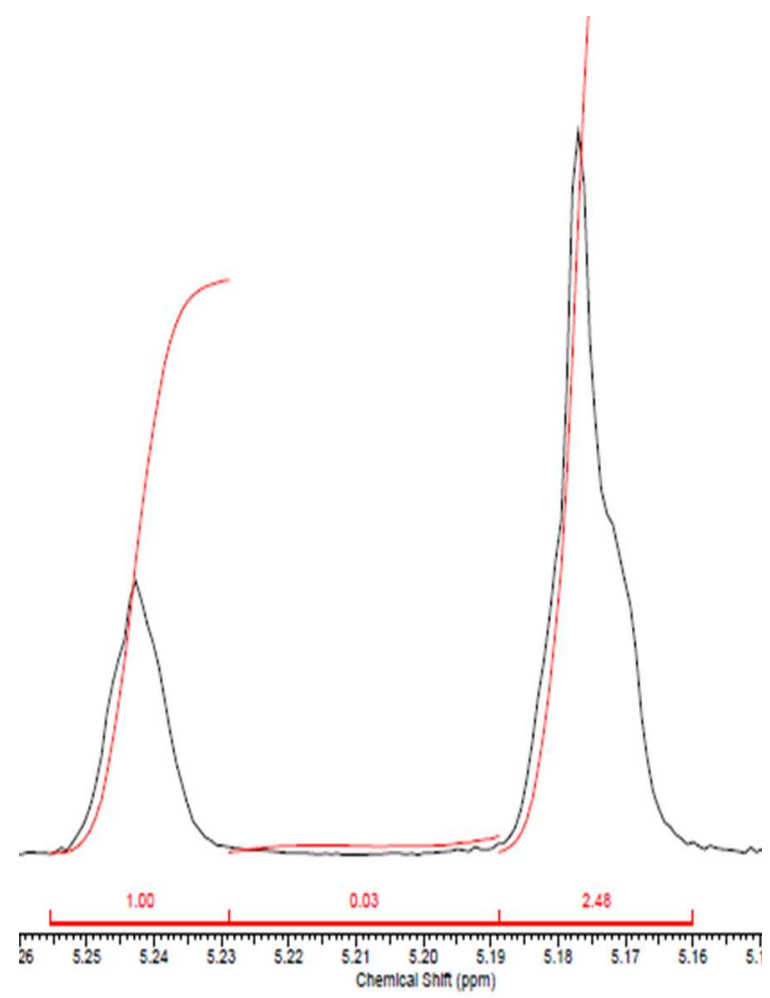

Figure S5. ${ }^{1} \mathrm{H}\left\{{ }^{1} \mathrm{H}\right\}$ NMR spectrum of polymer produced from reaction of $(R R / R R)-2$ with meso-lactide and calculation of $P_{s}$ value 


$$
\begin{aligned}
A & =(x+y+z) \\
& =5.5 \\
P r & =\left(\frac{2 x}{A}\right)^{\frac{1}{2}} \\
& =0.603 \\
P m & =\left(\frac{2 y}{A}\right) \div \operatorname{Pr} \\
= & 0.380
\end{aligned}
$$
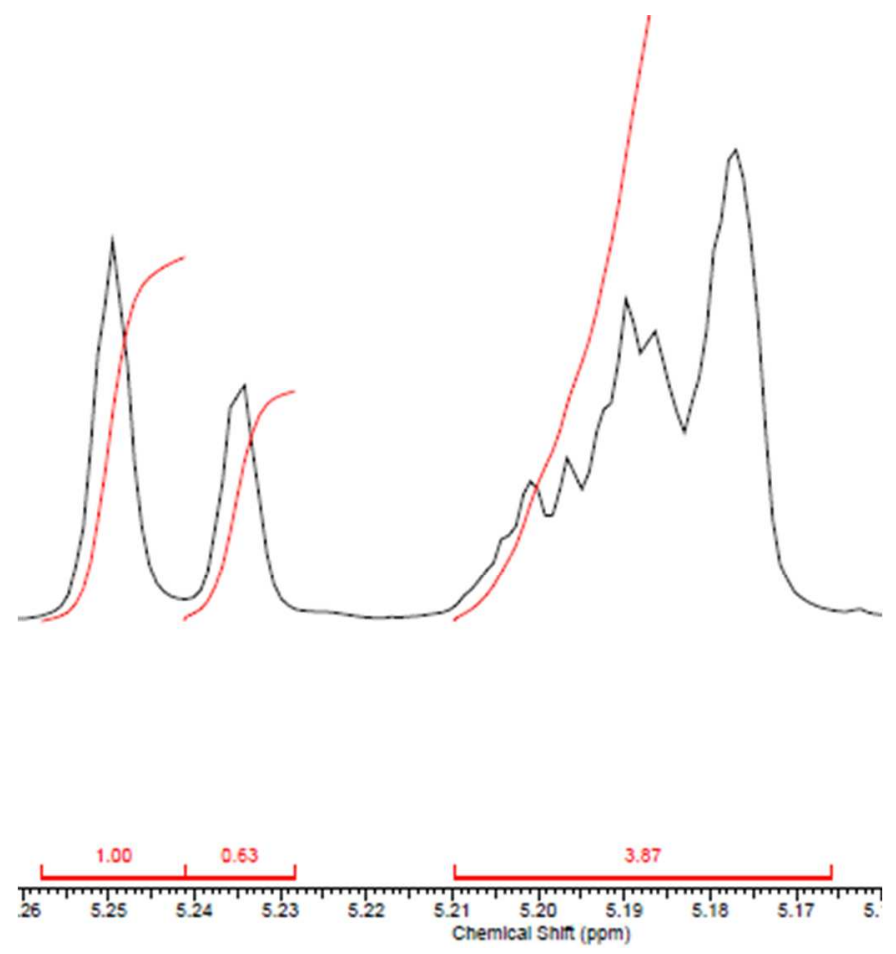

Figure S6. ${ }^{1} \mathrm{H}\left\{{ }^{1} \mathrm{H}\right\}$ NMR spectrum of polymer produced from reaction of tin octanoate and rac-lactide and calculation of $P_{r}$ value 


$$
\begin{aligned}
A & =(x+y+z) \\
& =2.19 \\
P r & =\left(\frac{2 \mathrm{x}}{\mathrm{A}}\right)^{\frac{1}{2}} \\
& =0.956 \\
P m & =\left(\frac{2 \mathrm{y}}{\mathrm{A}}\right) \div \operatorname{Pr} \\
& =0.067
\end{aligned}
$$

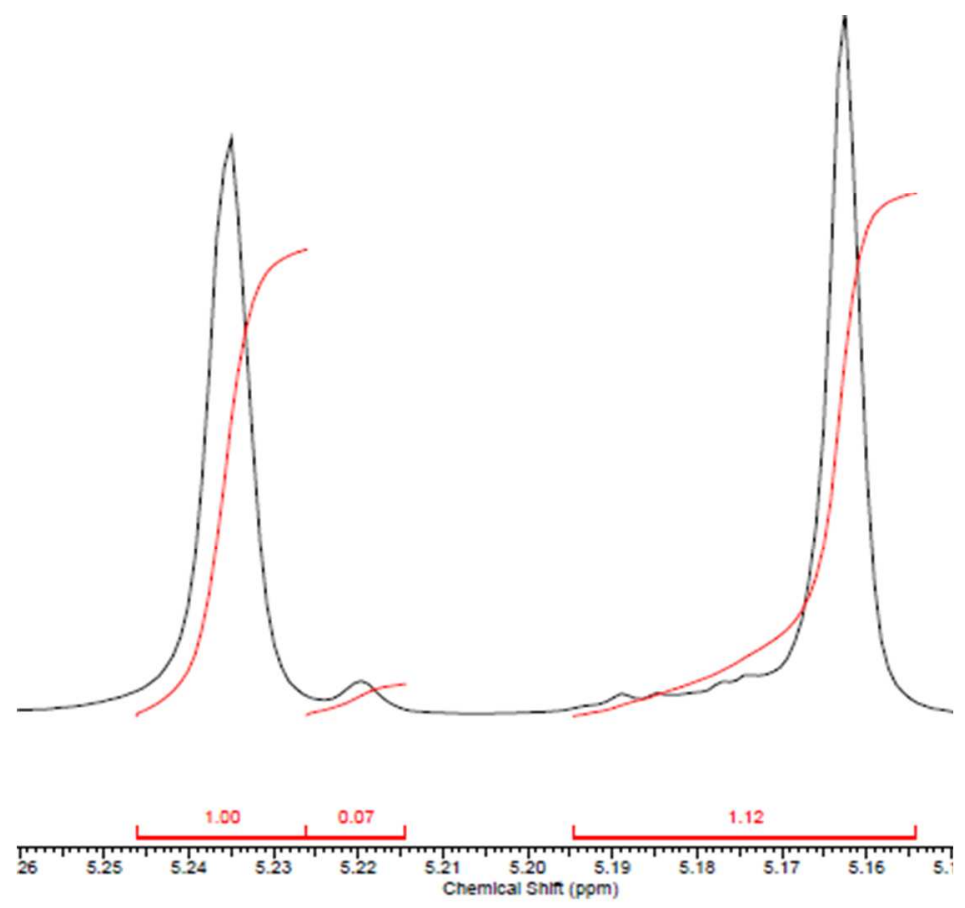

Figure S7. ${ }^{1} \mathrm{H}\left\{{ }^{1} \mathrm{H}\right\}$ NMR spectrum of polymer produced from reaction of $\mathrm{InCl}_{3} / \mathrm{BnOH} / \mathrm{NEt}_{3}$ and $r a c$-lactide and calculation of $P_{r}$ value 


$$
\begin{aligned}
A & =(x+y+z) \\
& =35.99
\end{aligned} \quad \begin{aligned}
P r & =\left(\frac{2 x}{A}\right)^{\frac{1}{2}} \\
& =0.236 \\
P m & =\left(\frac{2 y}{A}\right) \div \operatorname{Pr} \\
& =0.709
\end{aligned}
$$

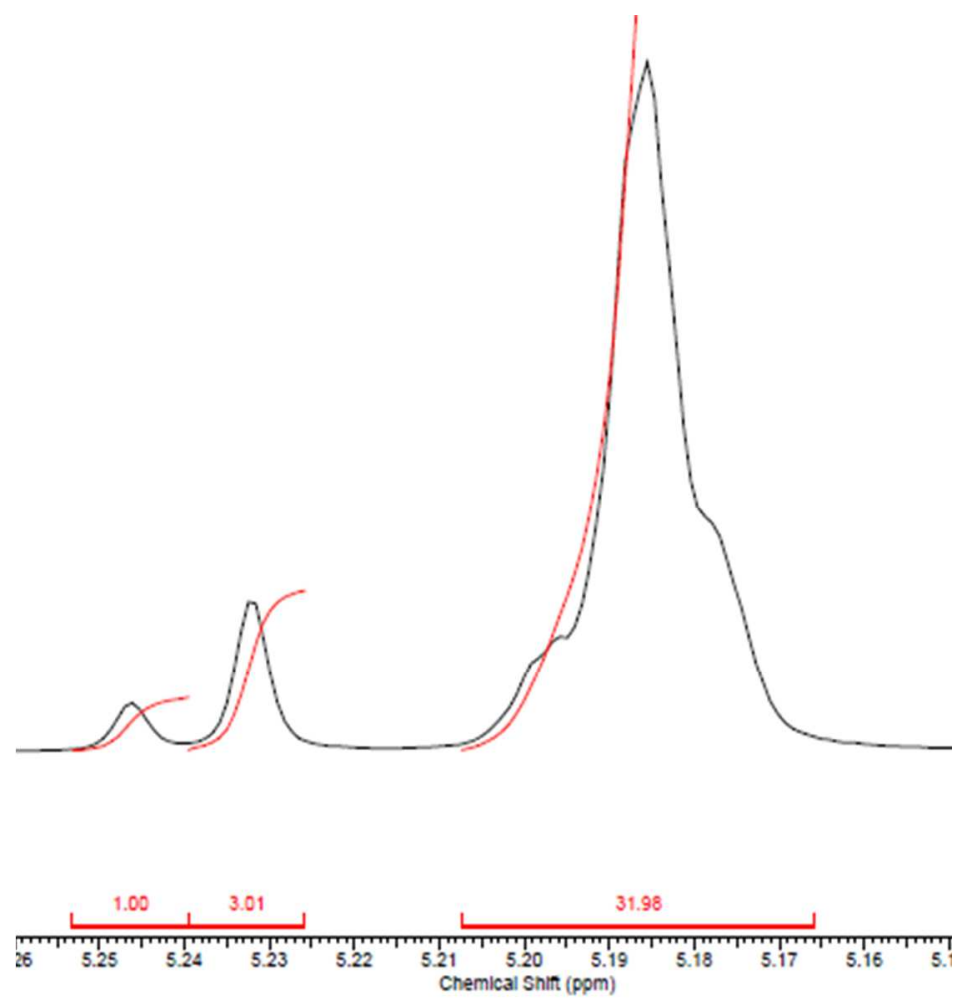

Figure S8. ${ }^{1} \mathrm{H}\left\{{ }^{1} \mathrm{H}\right\}$ NMR spectrum of polymer produced from reaction of $(R R / R R)-2$ with rac-lactide and calculation of $P_{s}$ value 


$$
\begin{aligned}
& \alpha=\frac{\mathrm{z}}{(\mathrm{x}+\mathrm{y}+\mathrm{z})} \\
& =0.867 \\
& {[\mathrm{sss}]=1 / 2\left[\alpha^{3}+(1-\alpha)^{3}+\alpha^{2}+(1-\alpha)^{2}\right]} \\
& =0.712 \\
& P \mathrm{i}=[\mathrm{sis}]=\alpha(1-\alpha) \\
& =0.115 \\
& {[\mathrm{ssi} / \text { iss } / \text { isi }]=3\left(\frac{1}{2}\left[\alpha^{2}(1-\alpha)+\alpha(1-\alpha)^{2}\right]\right)} \\
& =0.174 \\
& =0.886
\end{aligned}
$$

Without defect peaks

$P_{\mathrm{S}}=0.903$

Using peak intensities

$P_{S}=0.904$

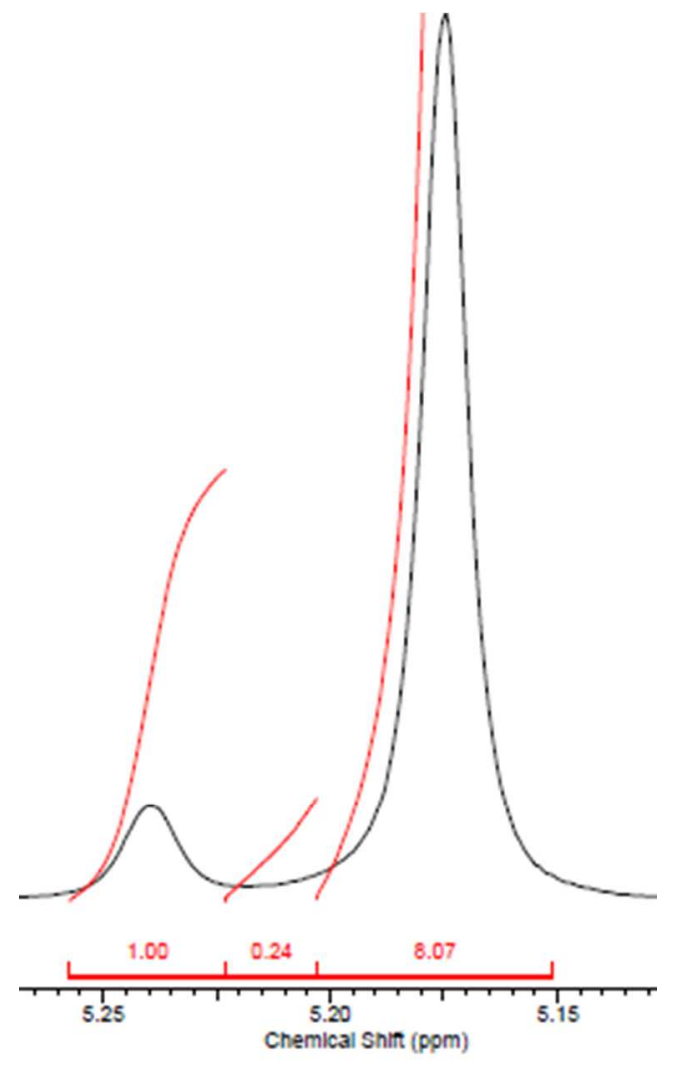

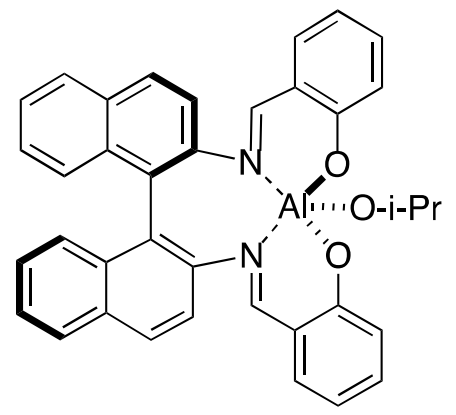

Figure S9. ${ }^{1} \mathrm{H} \quad\left\{{ }^{1} \mathrm{H}\right\}$ NMR spectrum of polymer produced from reaction of (-)-salen binathphylamine alkoxide complexes with meso-lactide and calculation of $P_{s}$ value 


\section{E. GCMS traces from meso-lactide purification}
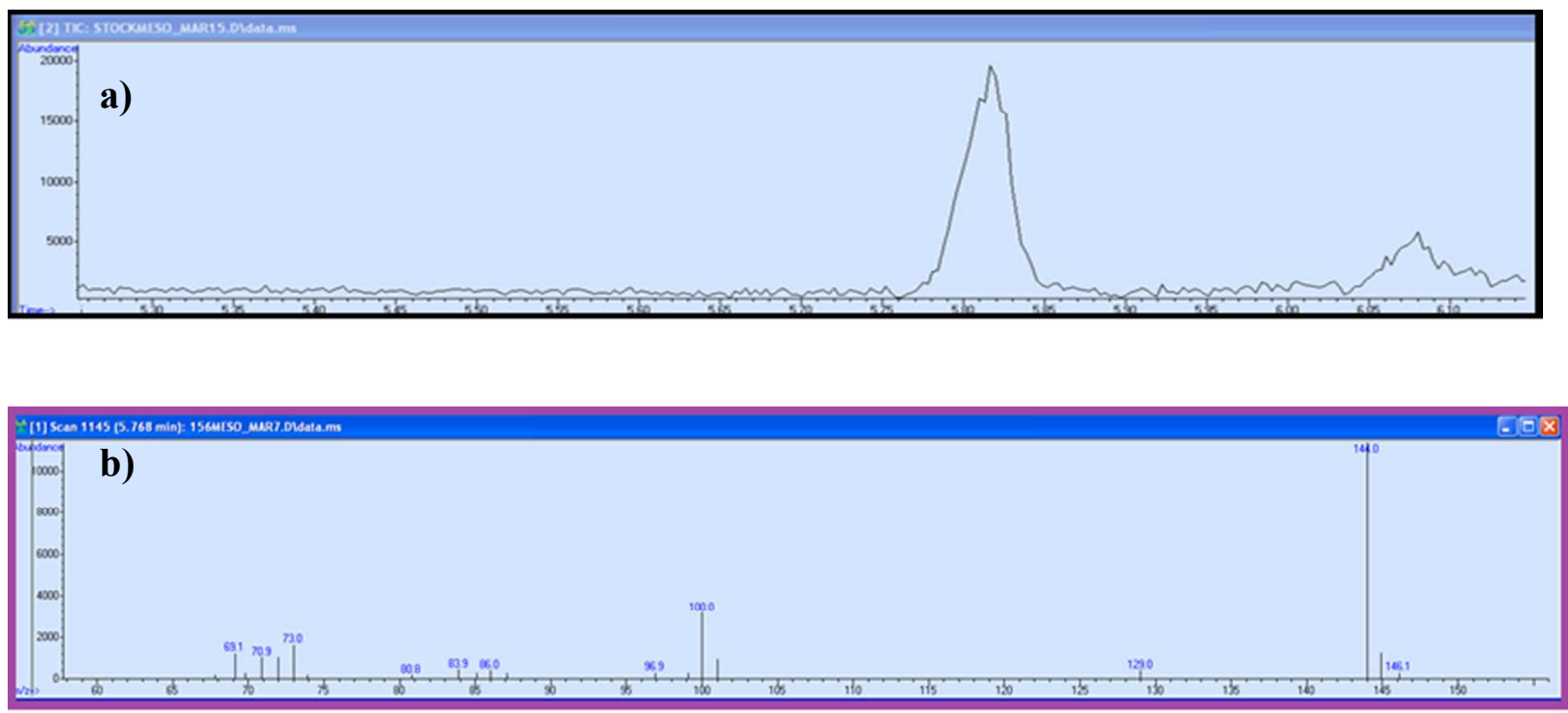

Figure S10. a) GCMS trace of meso-lactide before purification (26\% rac-lactide) b) mass spectral data for peak eluting at 5.90 minutes
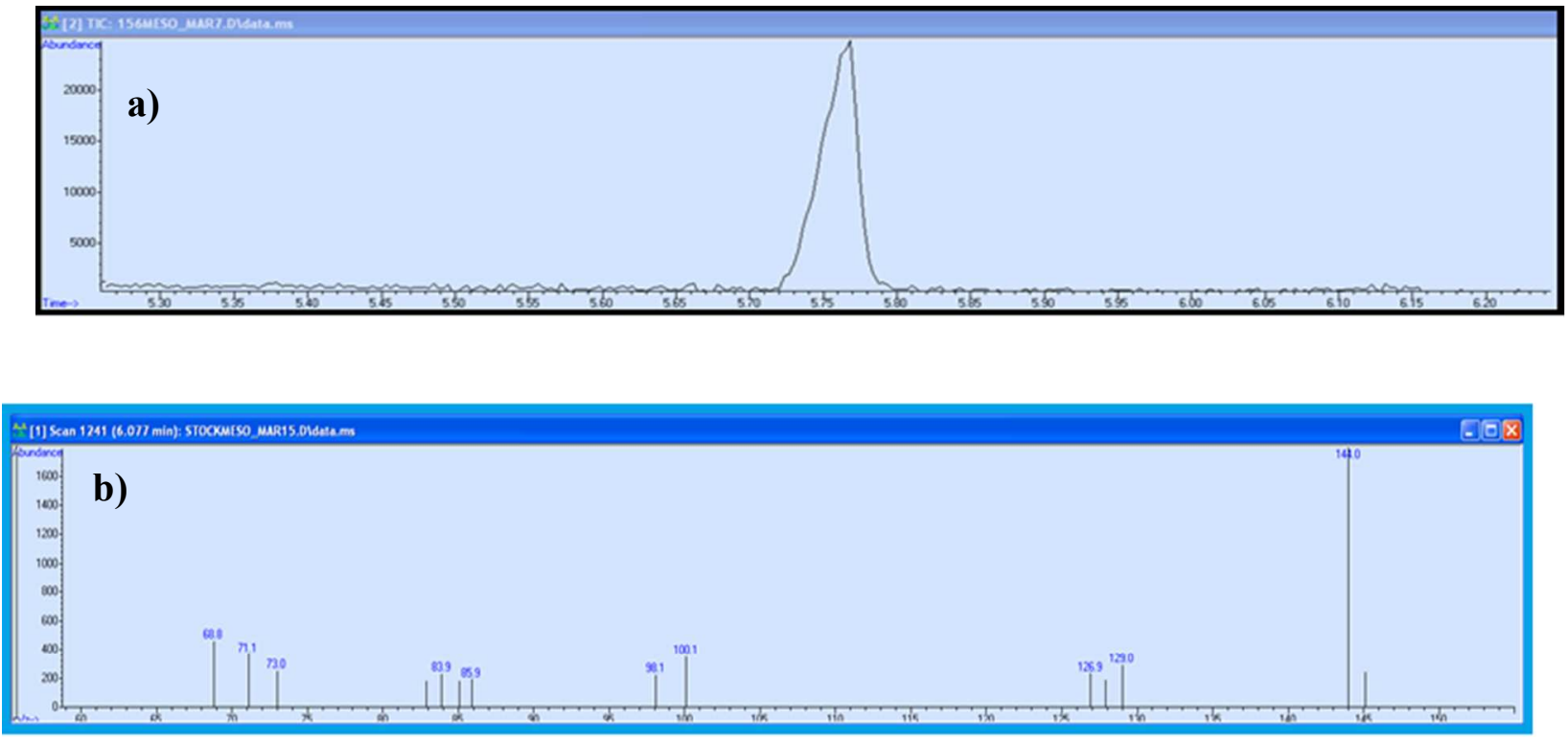

Figure S11. a) GCMS trace of meso-lactide after purification ( $<1 \% \mathrm{rac}$-lactide) b) mass spectral data for peak eluting at 5.75 minutes 


\section{F. TGA traces}

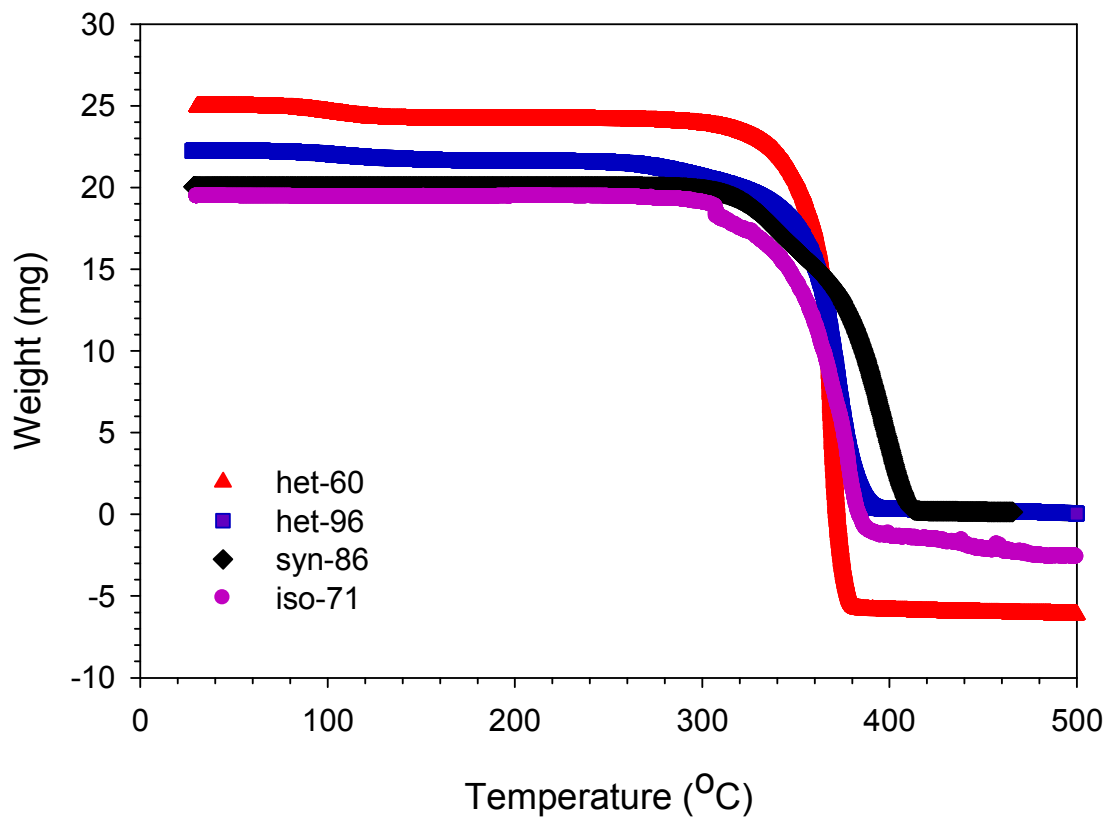

Figure S12. TGA heating traces for the various microstructured PLAs. Thermogravimetric analysis was performed on approximately $20 \mathrm{mg}$ of material. Samples were heated to $500{ }^{\circ} \mathrm{C}$ at a rate of $20^{\circ} \mathrm{C} / \mathrm{min}$ to determine the degradation onset temperature (temperature at which there is $5 \%$ weight loss), $\mathrm{T}_{5 \%}$. 


\section{G. Differential Scanning Calorimeter (DSC) traces.}

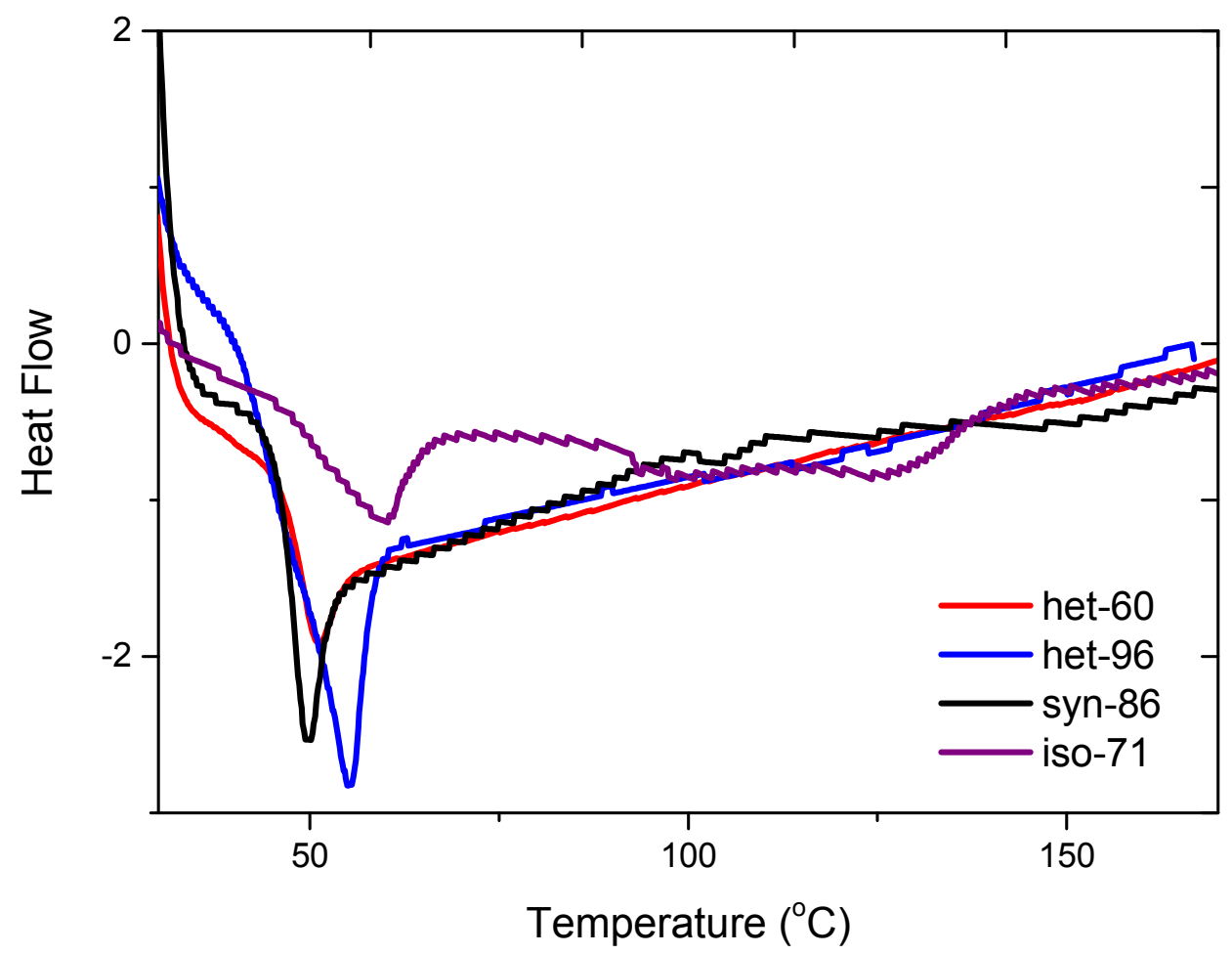

Figure S13. DSC traces for various microstructured PLAs studied. Samples heated to $170{ }^{\circ} \mathrm{C}$ at $10{ }^{\circ} \mathrm{C} / \mathrm{min}$ and cooled to $25^{\circ} \mathrm{C}$ at $5{ }^{\circ} \mathrm{C} / \mathrm{min}$ under a nitrogen atmosphere to reduce sample degradation. Glass transition and melting temperatures calculated from first heating scans. $c a .2$ mg of sample used. 


\section{H. Isothermal Time Sweep Experiments}

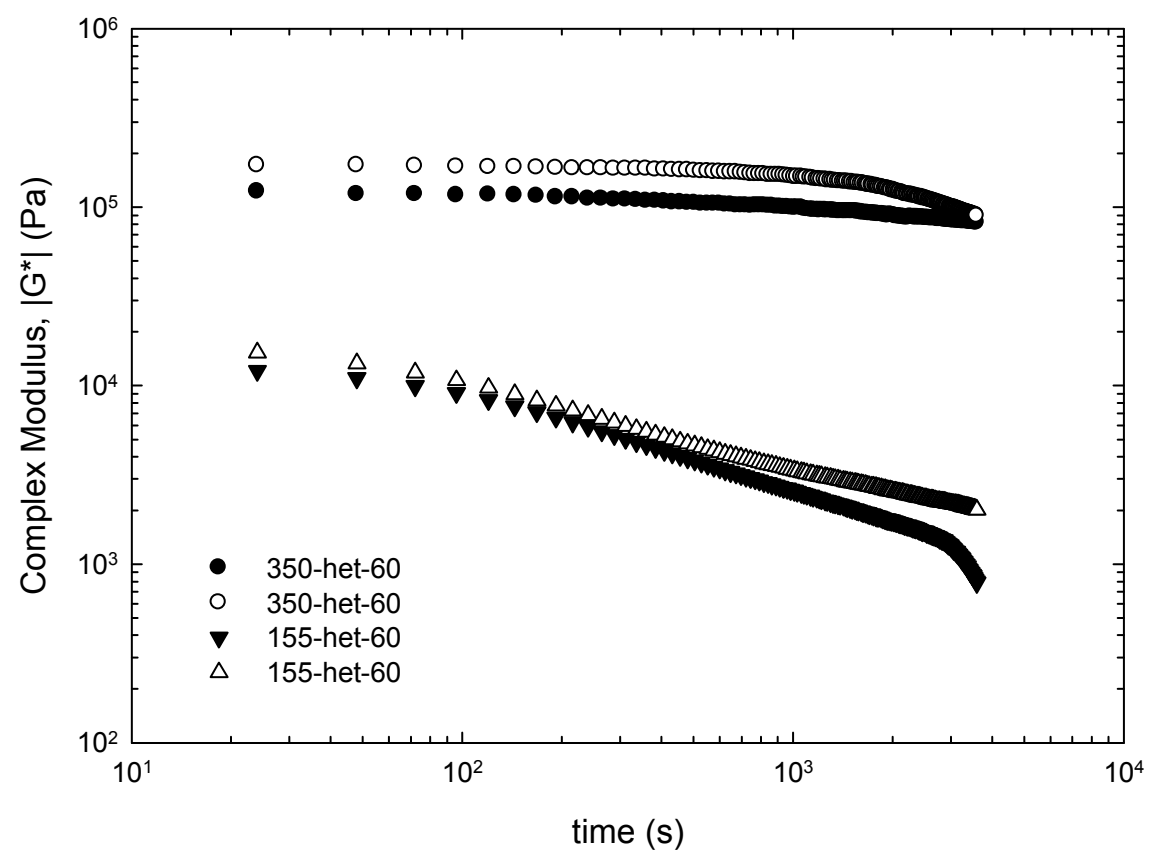

Figure S14. Complex modulus, $\left|\mathrm{G}^{*}\right|$, vs. time for het-60 polymers of various molecular weights. 


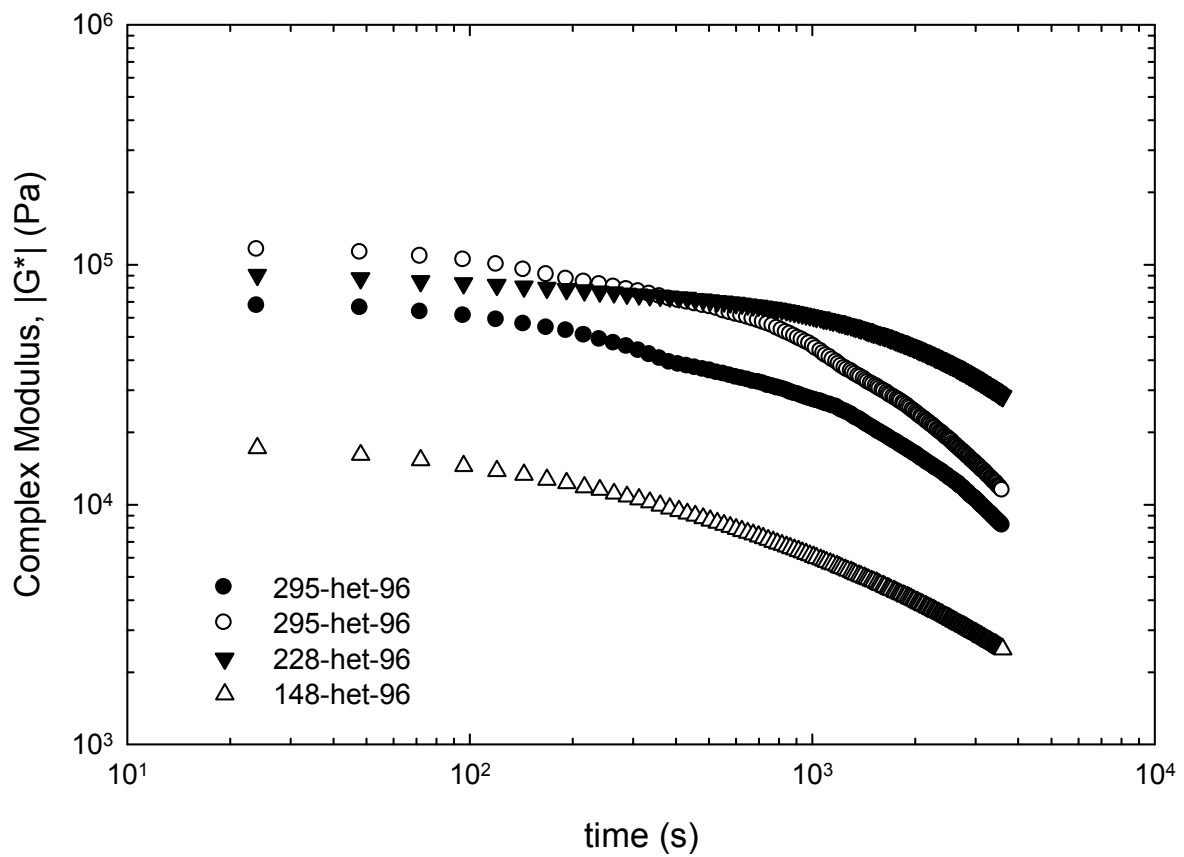

Figure S15. Complex modulus, $\left|G^{*}\right|$, vs. time for het-96 polymers of various molecular weights.

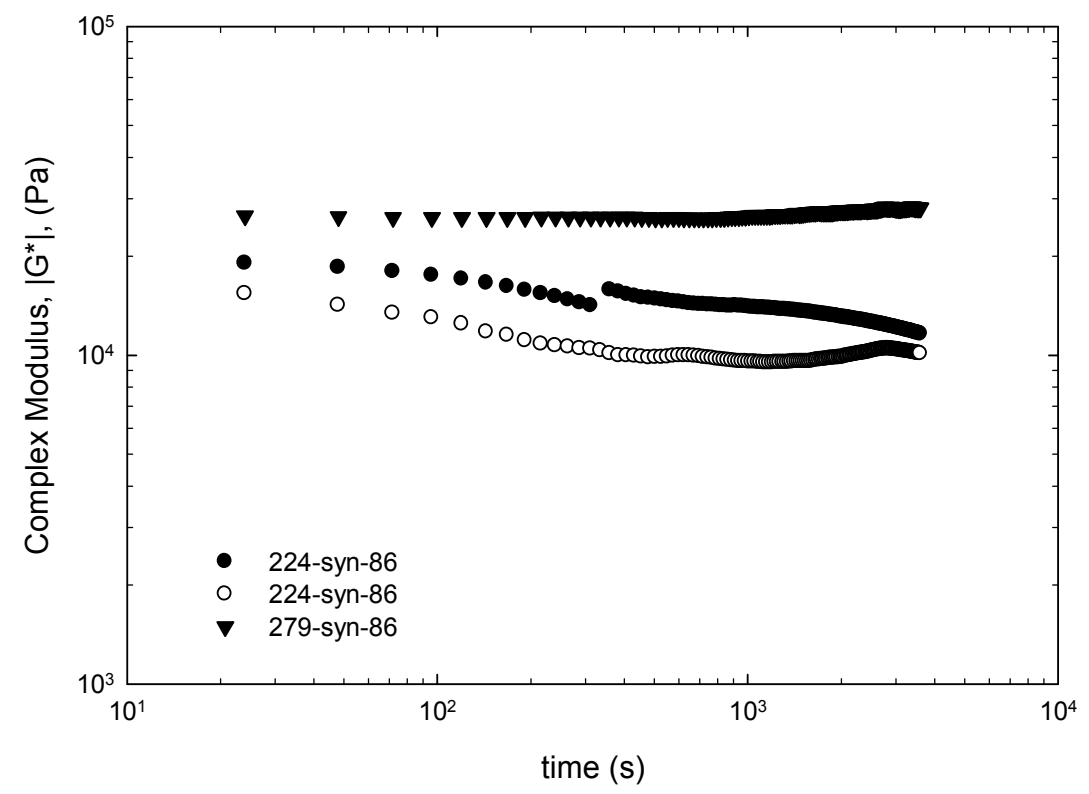

Figure S16. Complex modulus, $\left|G^{*}\right|$, vs. time for syn- 86 polymers of various molecular weights. 


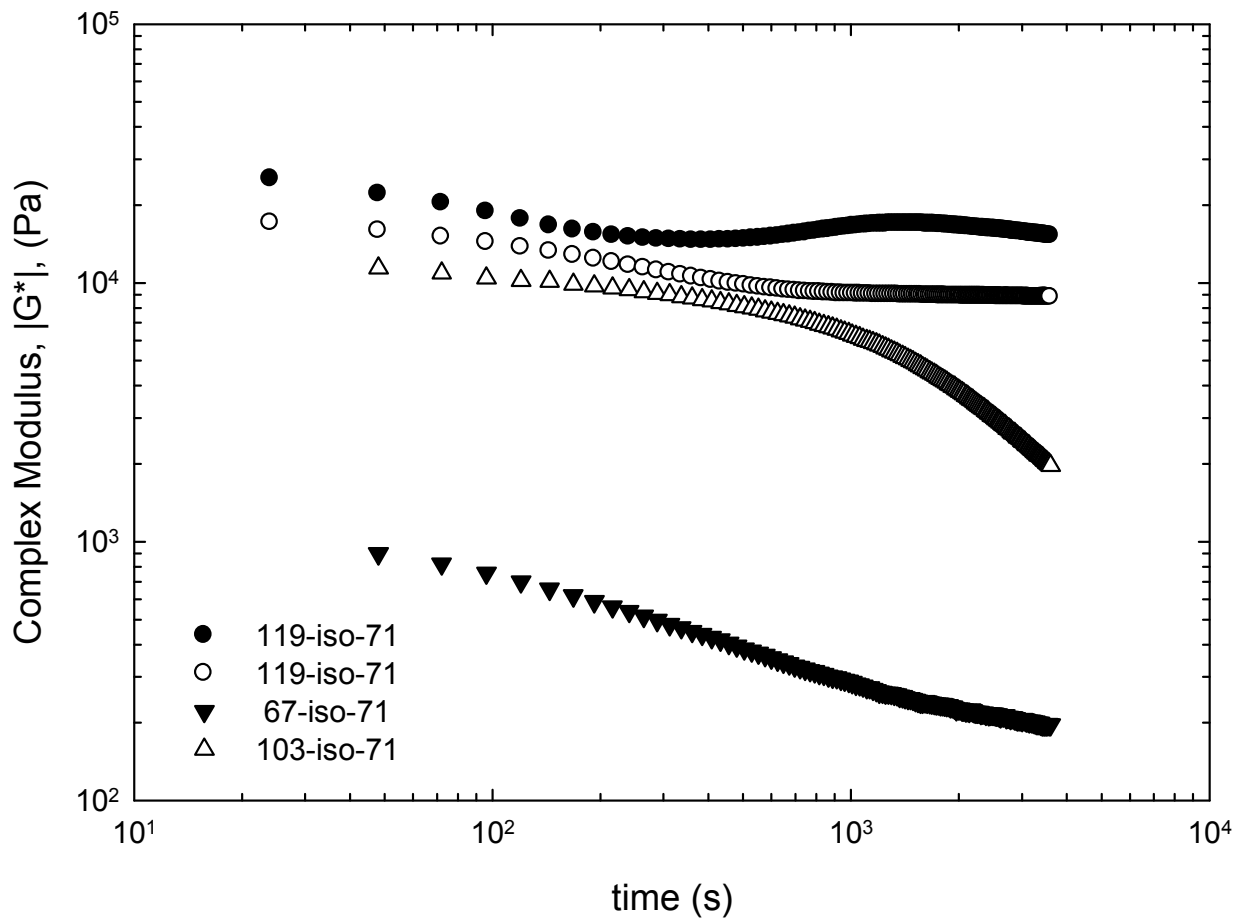

Figure S17. Complex modulus, $\left|\mathrm{G}^{*}\right|$, vs. time for iso-71 polymers of various molecular weights. 


\section{Van Gurp-Palmen plots}

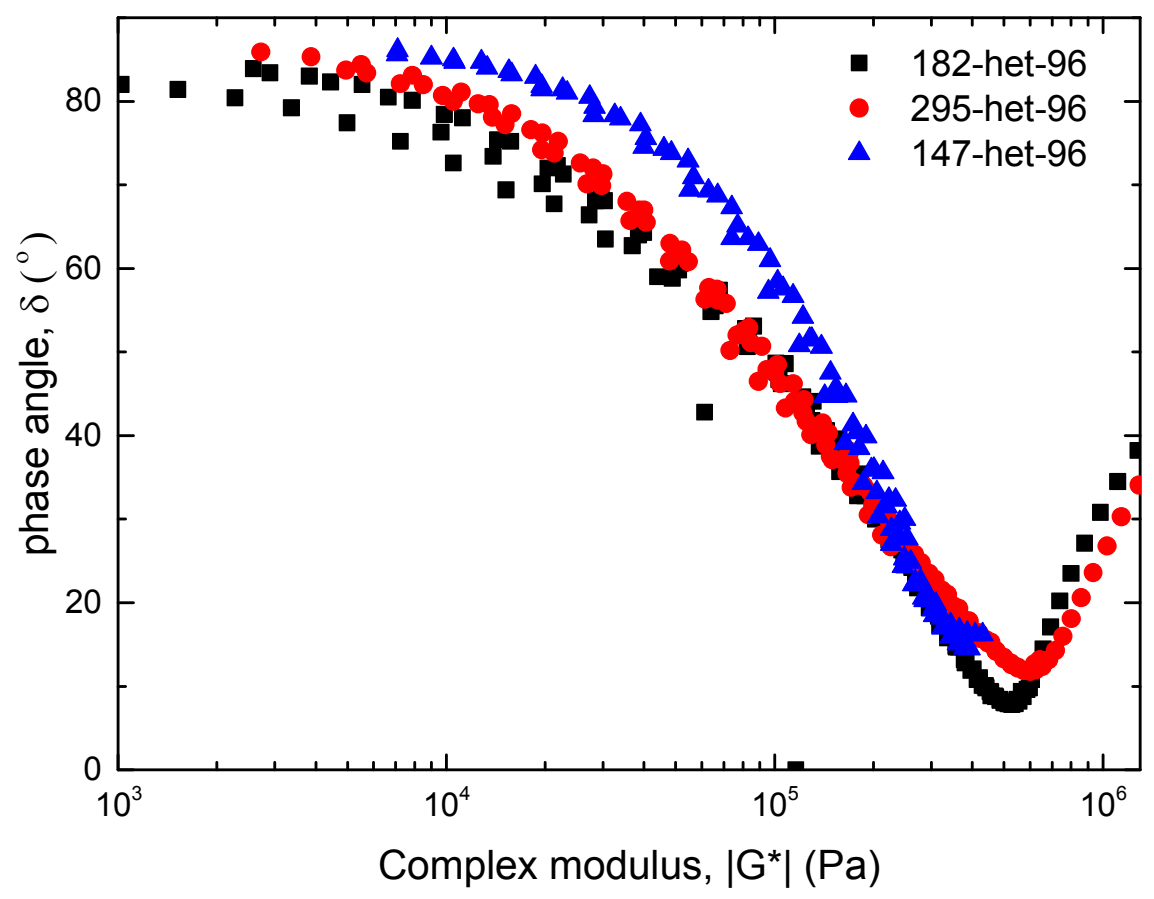

Figure S18. Van Gurp-Palmen plots of het-96 polymers with varying molecular weights. 


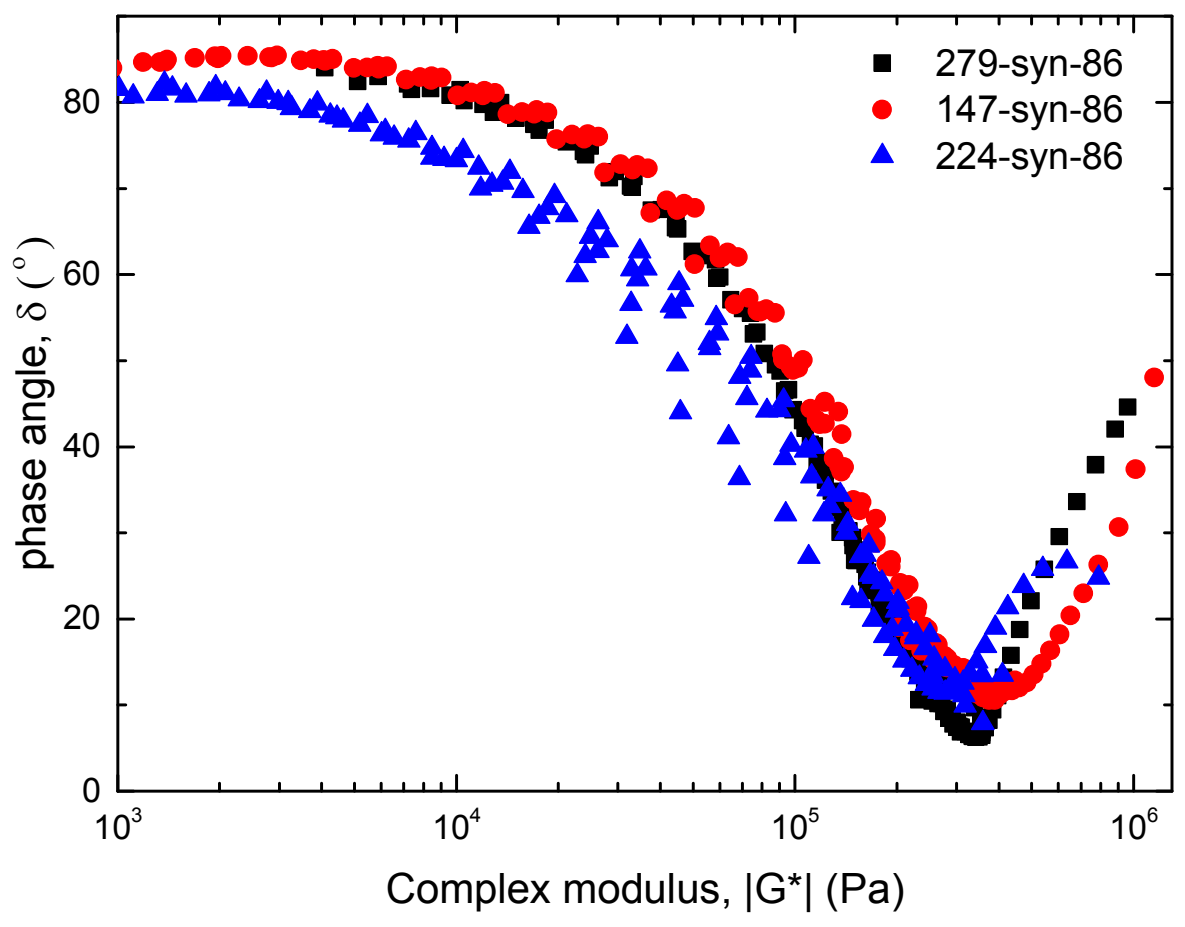

Figure S19. Van Gurp-Palmen plots of syn-86 polymers with varying molecular weights. 


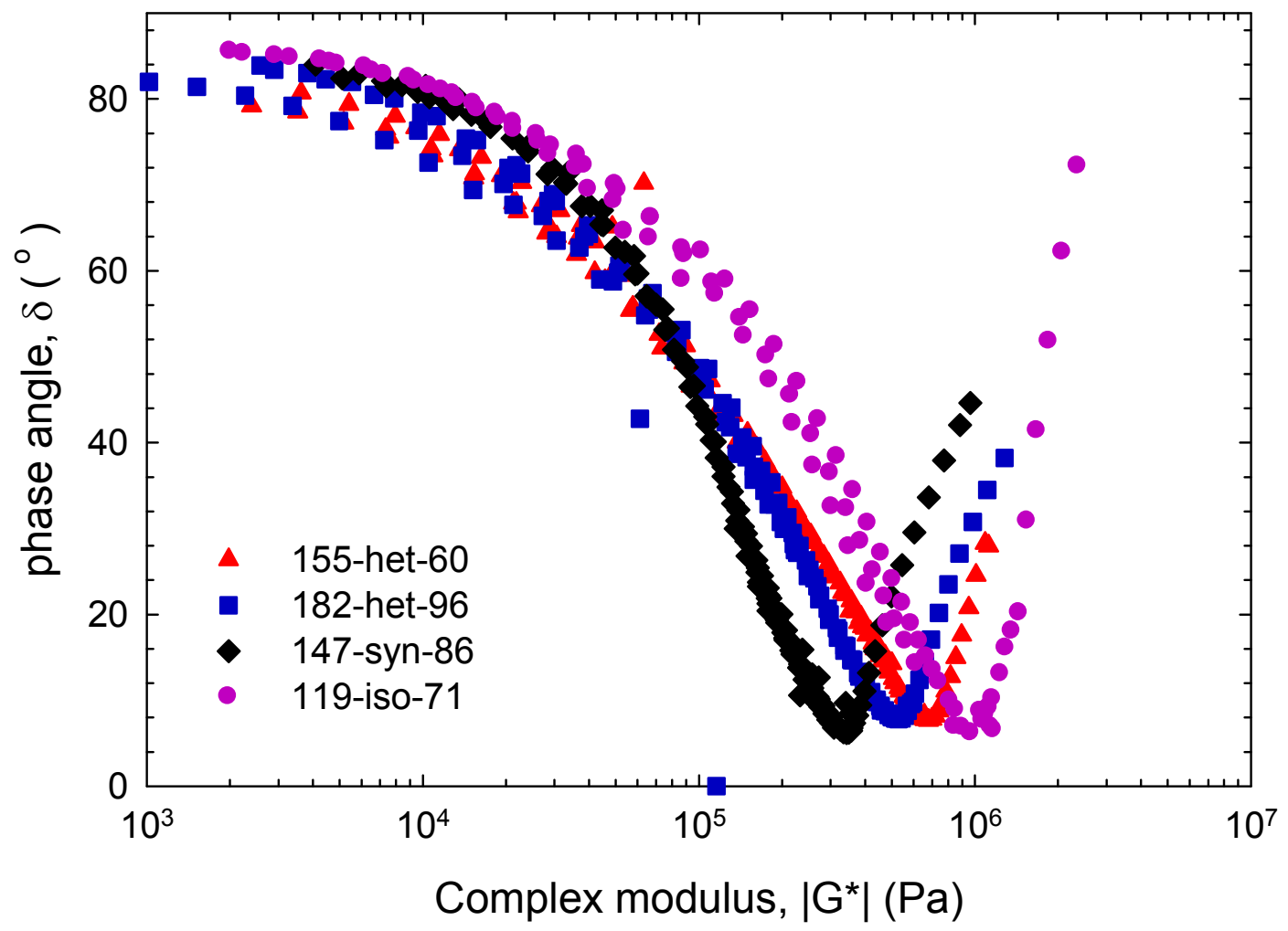

Figure S20. Van Gurp-Palmen plots of the various microstructured PLAs 


\section{J. Arrhenius plots for calculation of flow activation energies}

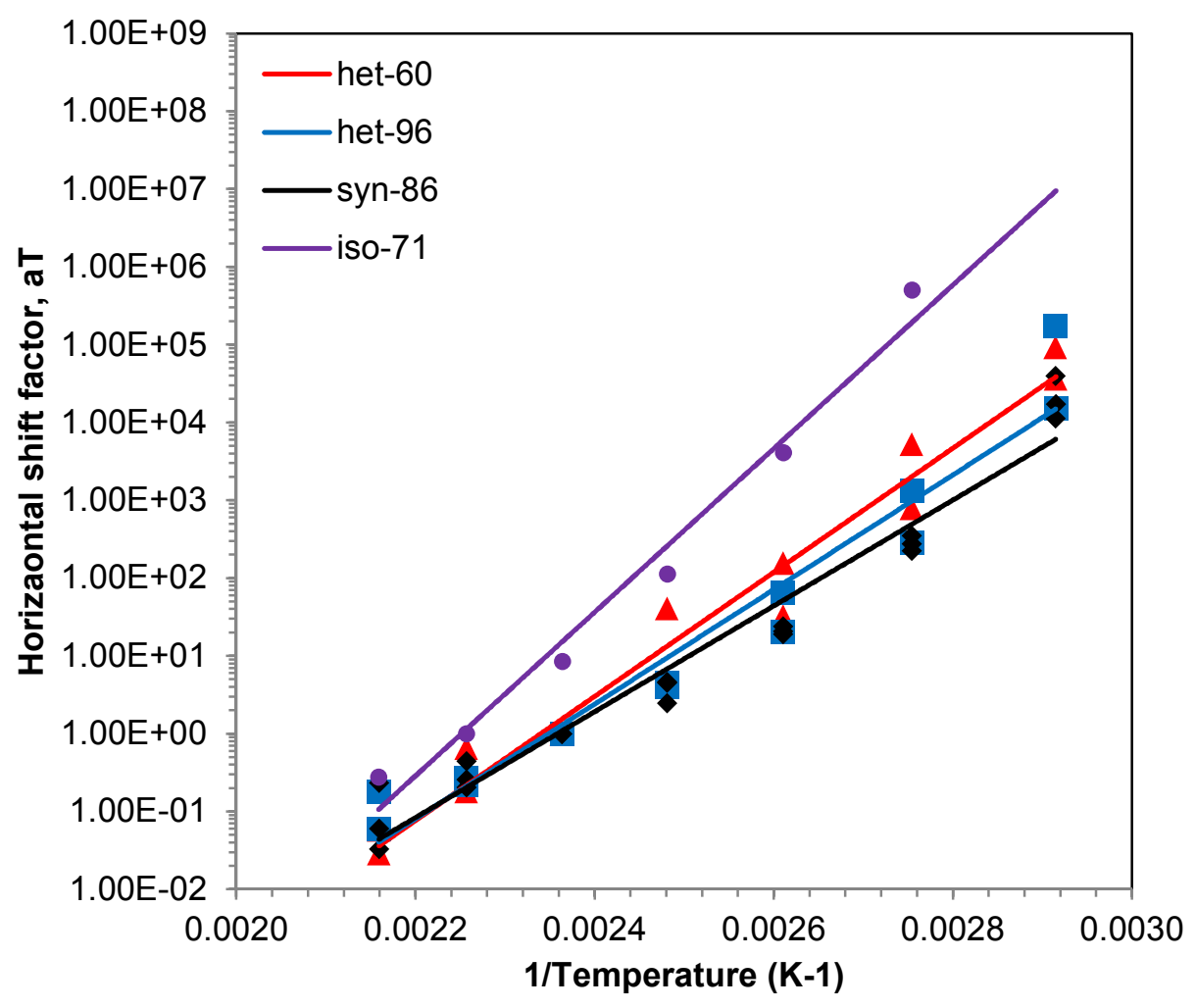

Figure S21. The horizontal shift factors, $\mathrm{a}_{\mathrm{T}}$ for PLAs listed in Table I at the reference temperature of $150{ }^{\circ} \mathrm{C}$. Lines represents single fitting of the data used to calculate flow activation energy, $E_{a, f l o w}$. 


\section{K. Uniaxial Extensional Experimental Plots}
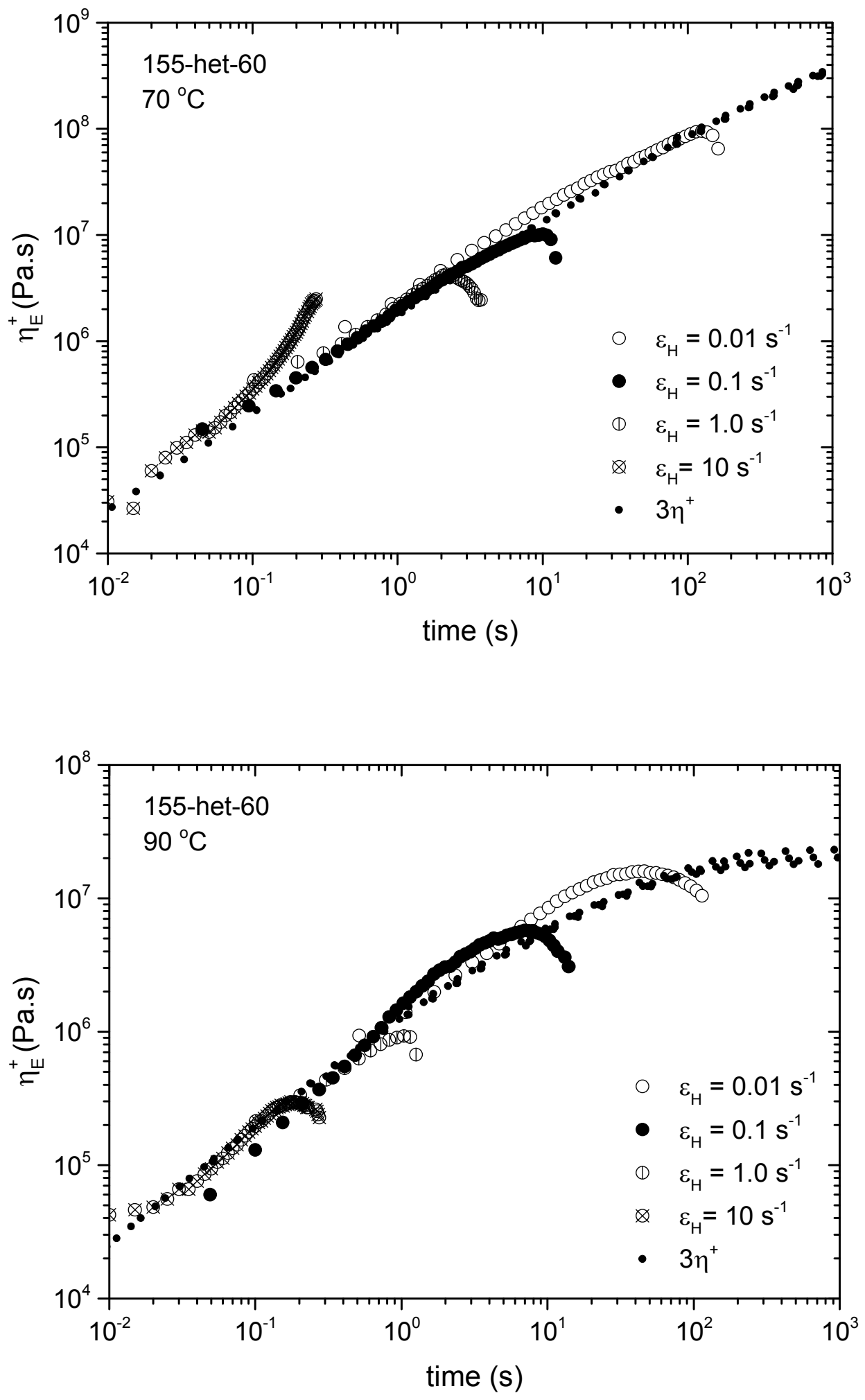


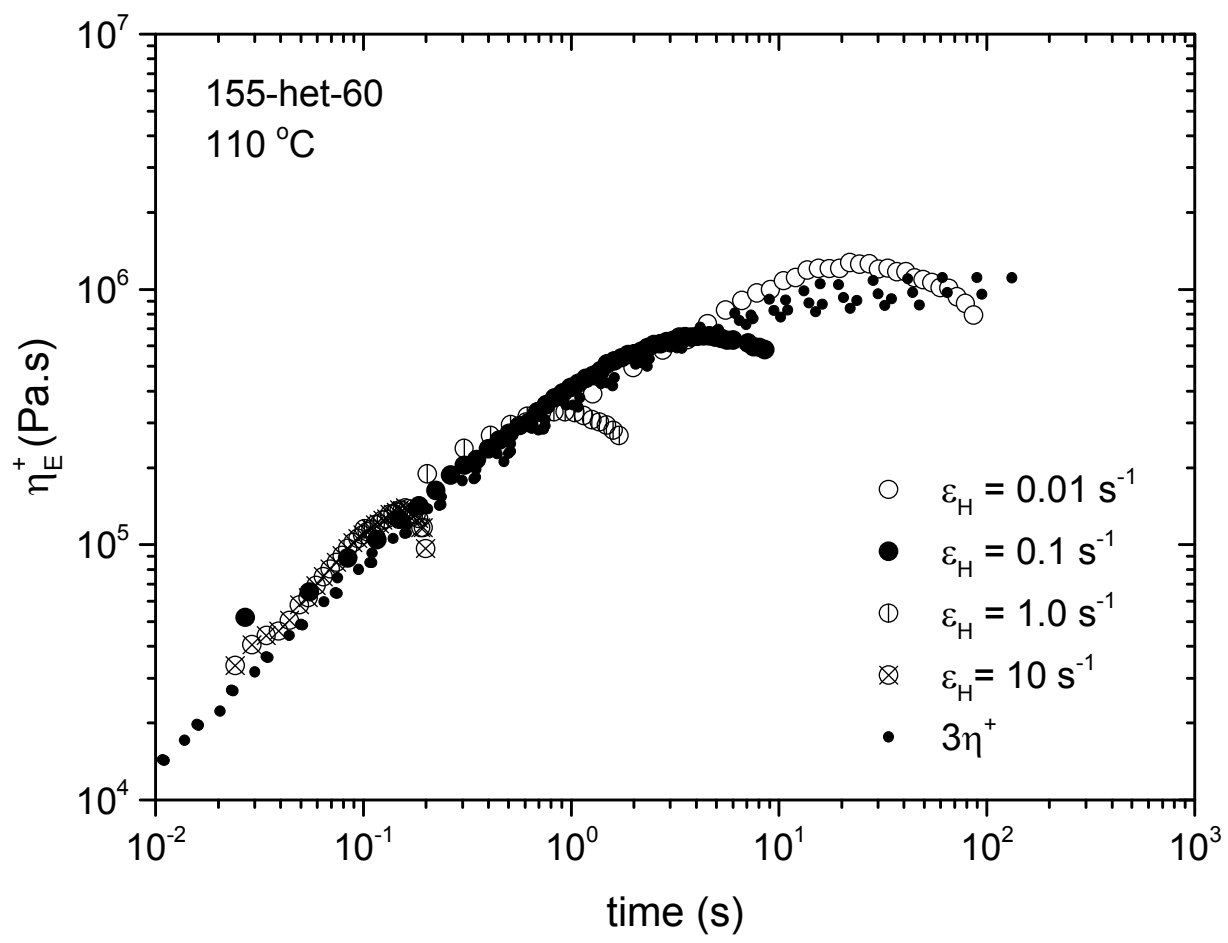

Figure S22. Elongational viscosity as a function of time at Hencky strain rates from 0.01 to $10 \mathrm{~s}^{-1}$ for (Table 3, entry 1). 

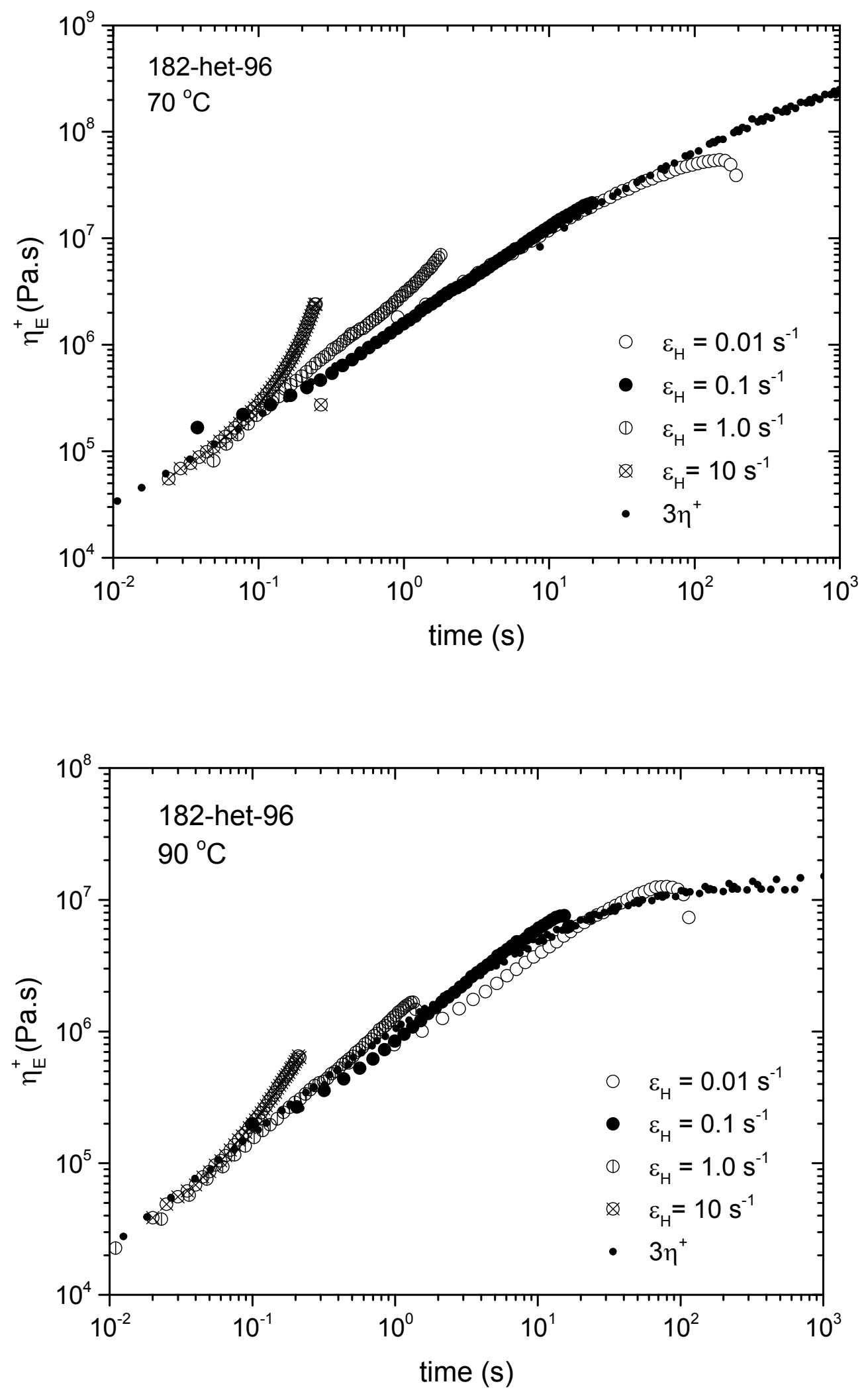


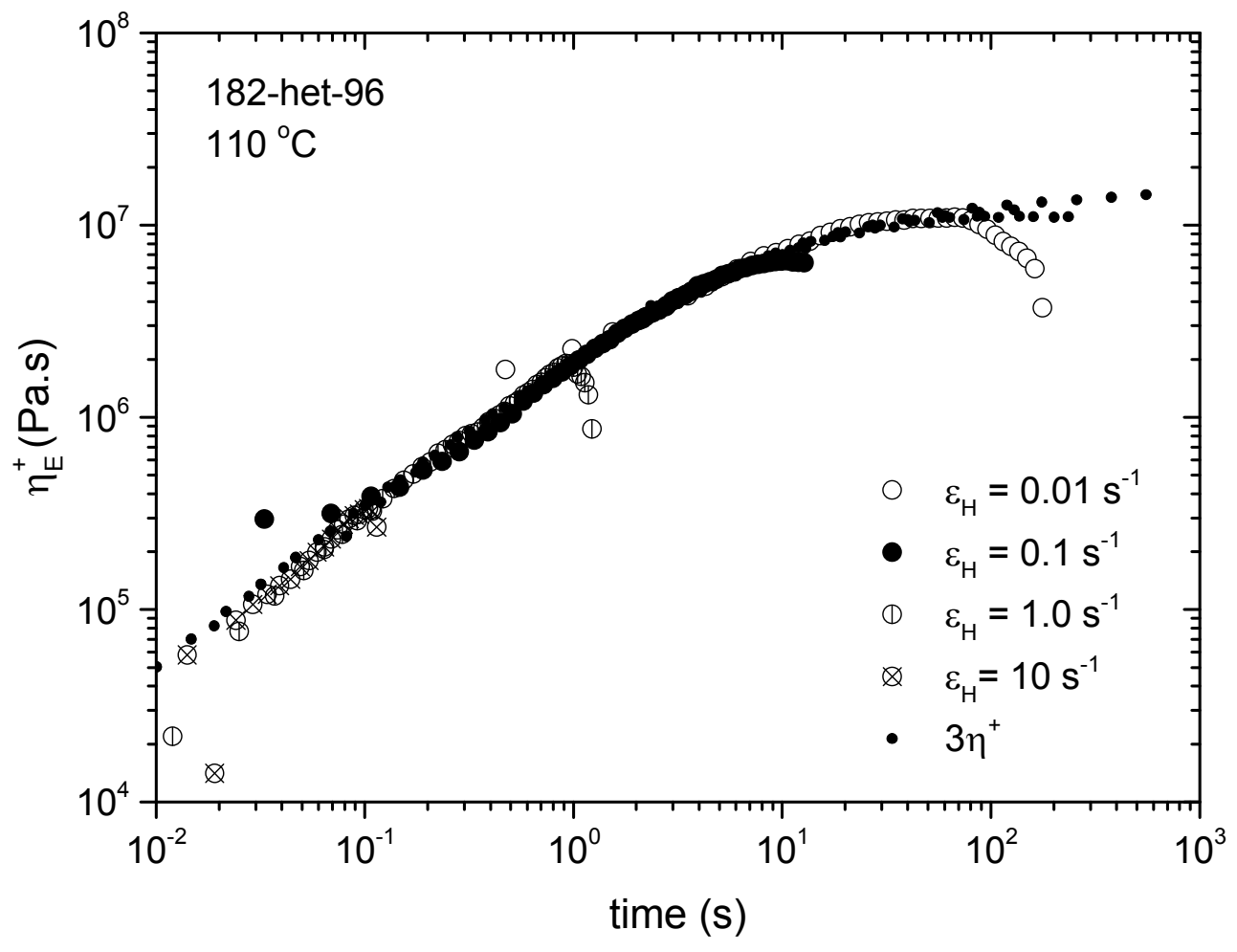

Figure S23. Elongational viscosity as a function of time at Hencky strain rates from 0.01 to $10 \mathrm{~s}^{-1}$ for heterotactic PLA (Table 3, entry 2). 

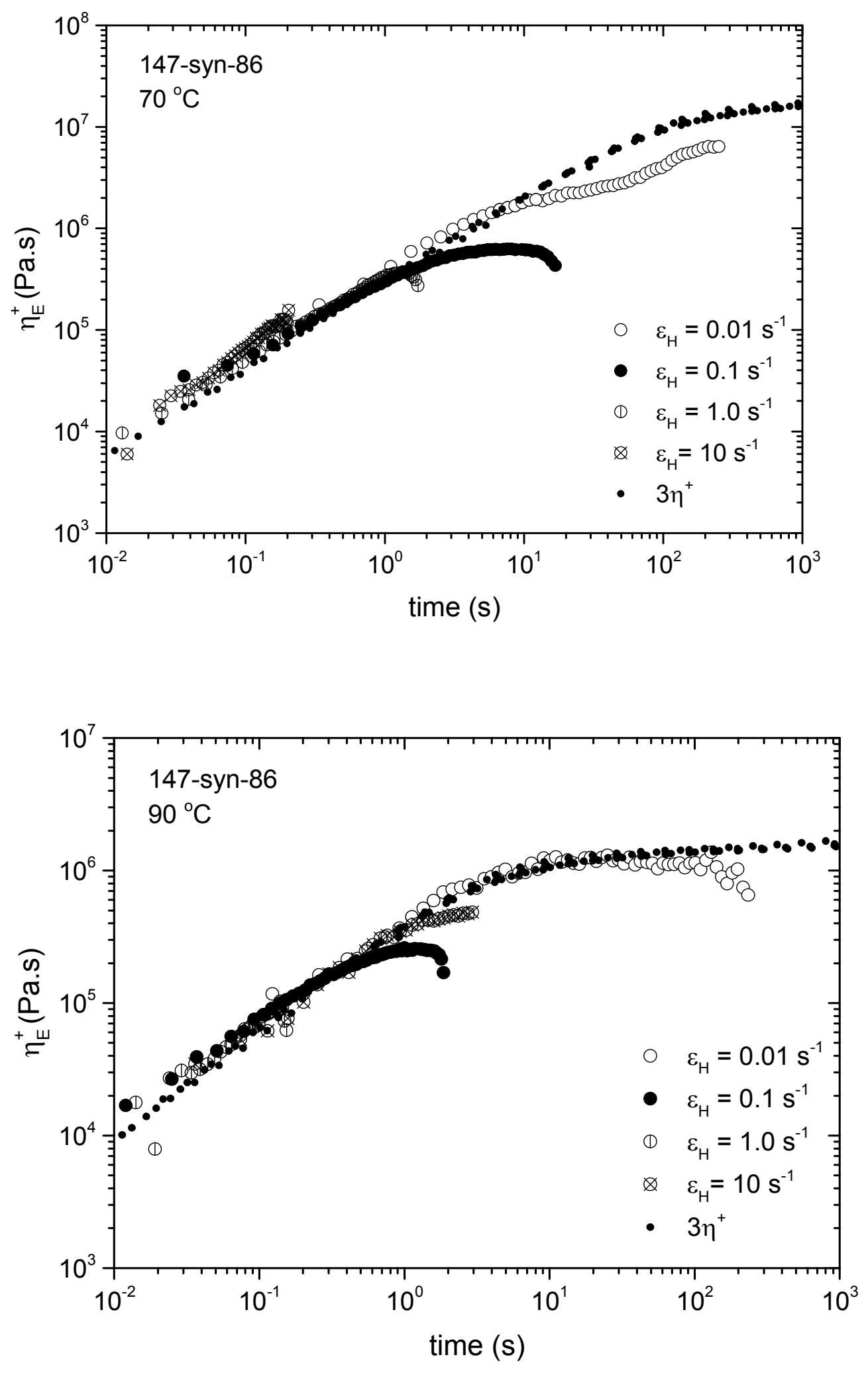


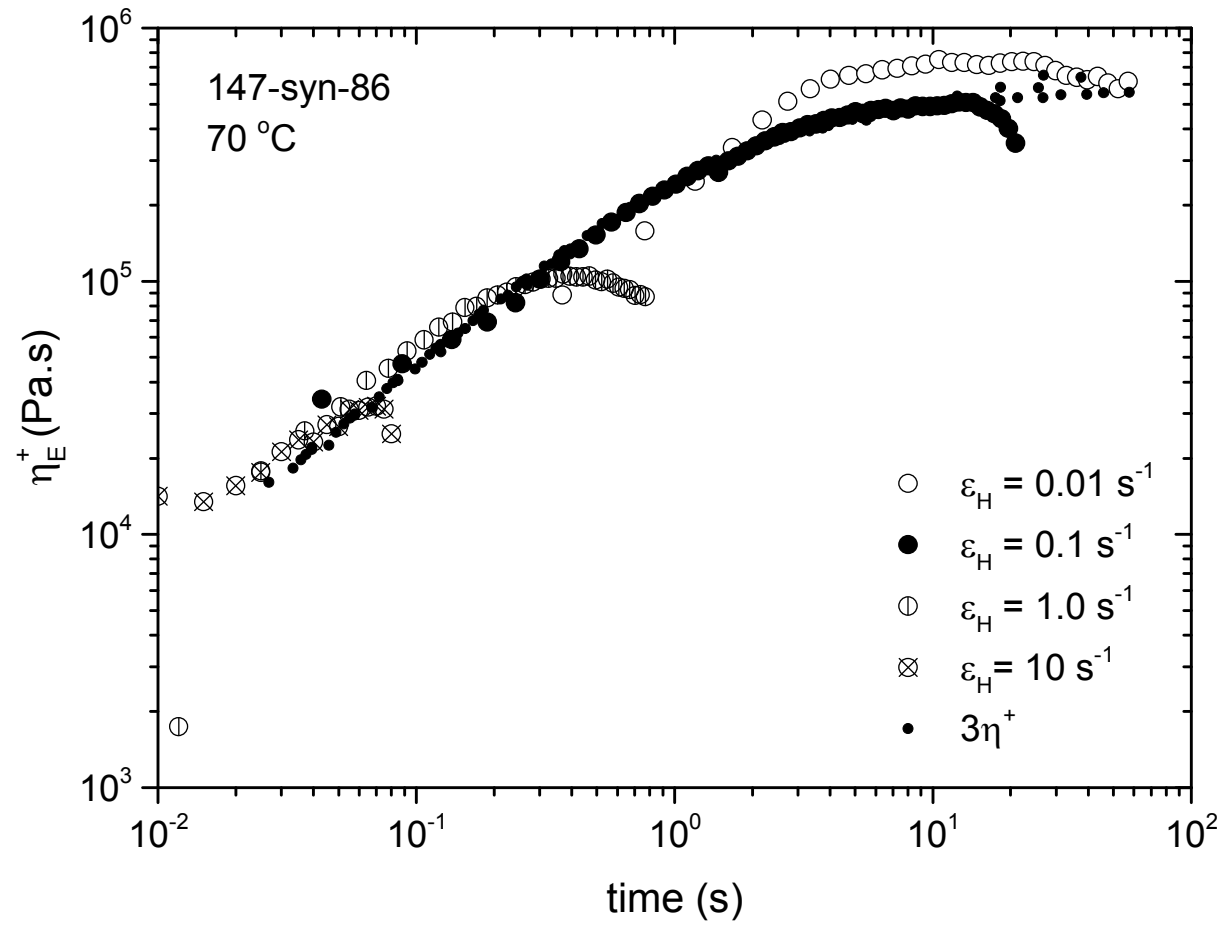

Figure S24. Elongational viscosity as a function of time at Hencky strain rates from 0.01 to $10 \mathrm{~s}^{-1}$ for syndio-enriched PLA (Table 3, entry 3). 

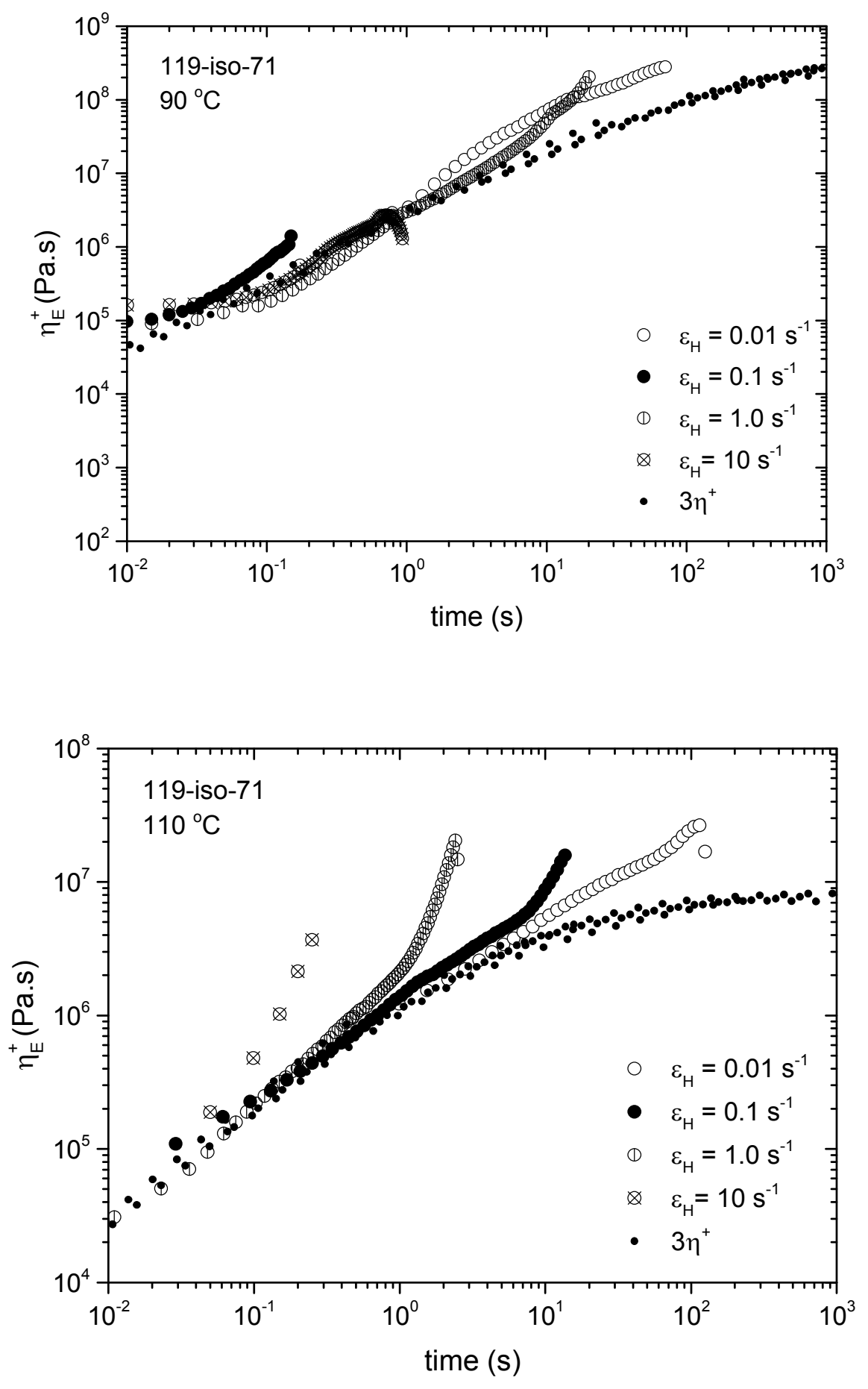

Figure S25. Elongational viscosity as a function of time at Hencky strain rates from 0.01 to $10 \mathrm{~s}^{-1}$ for iso-enriched gradient PLA (Table 3, entry 4). Uniaxial elongation experiments on iso-70 were unable to be performed at $70{ }^{\circ} \mathrm{C}$ due to instrument constraints. 


\section{References}

1. (a) Zell, M. T.; Padden, B. E.; Paterick, A. J.; Thakur, K. A. M.; Kean, R. T.; Hillmyer, M. A.; Munson, E. J., Macromolecules. 2002, 35, 7700; (b) Thakur, K. A. M.; Kean, R. T.; Zell, M. T.; Paddenb, E. B.; Munson, E. J., Chem. Commun. 1998, 1913; (c) Thakur, K. A. M.; Kean, R. T.; Hall, E. S.; Kolstad, J. J.; Munson, E. J., Macromolecules. 1998, 31, 1487-1494; (d) Thakur, K. A. M.; Kean, R. T.; Hall, E. S.; Kolstad, J. J.; Lindgren, T. A., Macromolecules. 1997, 30, 2422-2428; (e) Chisholm, M. H.; Iyer, S. S.; McCollum, D. G.; Pagel, M.; WernerZwanziger, U., Macromolecules. 1999, 32, 963-973; (f) Chisholm, M. H.; Iyer, S. S.; Matison, M. E.; McCollum, D. G.; Pagel, M., Chem. Commun. 1997, 1999-2000; (g) Chisholm, M. H.; Gallucci, J.; Phomphrai, K., Chem. Commun. 2003, 48-49; (h) Coudane, J.; Ustarez-Peyret, C.; Schwach, G.; Vert, M., J. Poly. Sci. A. 1997, 35, 1651-1658; (i) Kricheldorf, H. R.; Boettcher, C.; Tonnes, K., Polymer. 1992, 33, 2817; (j) Kricheldorf, H. R.; Boettcher, C.; Tonnes, K., Polymer. 1992, 33, 2817-2824; (k) Kasperczyk, J.; Bero, M., Polymer. 2000, 41, 391-395.

2. When discussing meso-LA, literature conventions use s/i notation where s denotes a syndio linkage where the lactyl units have opposite configuration and i denotes an iso linkage where two bound units have the same arrangement and so tacticities are reported as Pi and Ps values (the probability of having iso linkages or syndio linkages within the polymer chain respectively).

3. Buffet, J.-C.; Okuda, J., Polym. Chem. 2011, 2, 2758-2763.

4. Ovitt, T. M.; Coates, G. W., J. Am. Chem. Soc. 2002, 124, 1316-1326. 
5. (a) Thakur, K. A. M.; Kean, R. T.; Zell, M. T.; Padden, B. E.; Munson, E. J., Chem. Commun. 1998, 1913-1914; (b) Zell, M. T.; Padden, B. E.; Paterick, A. J.; Thakur, K. A. M.;

Kean, R. T.; Hillmyer, M. A.; Munson, E. J., Macromolecules. 2002, 35, 7700-7707.

6. Yu, I.; Acosta-Ramirez, A.; Mehrkhodavandi, P., J. Am. Chem. Soc. 2012, 134, 1275812773.

7. Aluthge, D. C.; Patrick, B. O.; Mehrkhodavandi, P., Chem. Commun. 2013, 49, 4295.

8. $\quad$ Buffet, J. C.; Okuda, J., Chem .Commun. 2011, 47, 4796-4798.

9. Du, H.; Velders, A. H.; Dijkstra, P. J.; Zhong, Z.; Chen, X.; Feijen, J., Macromolecules. 2009, 42, 1058-1066.

10. Buffet, J.-C.; Martin, A. N.; Kol, M.; Okuda, J., Polymer Chemistry. 2011, 2, 2378. 